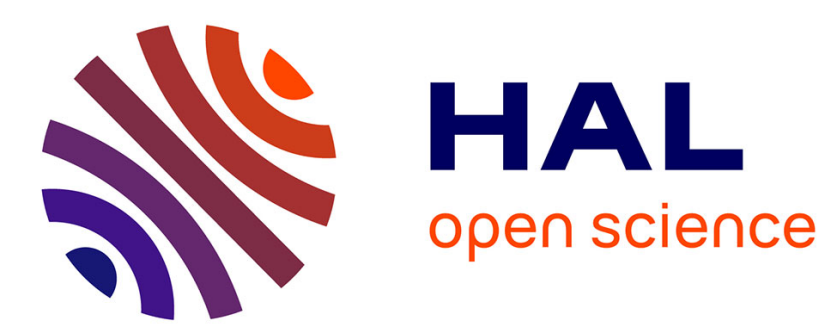

\title{
Building strategies to ensure langage coexistence in presence of bilingualism
}

\author{
C. Bernard, S. Martin
}

\section{To cite this version:}

C. Bernard, S. Martin. Building strategies to ensure langage coexistence in presence of bilingualism. Applied Mathematics and Computation, 2012, 218 (17), p. 8825 - p. 8841. 10.1016/j.amc.2012.02.041 . hal-00766149

HAL Id: hal-00766149

https://hal.science/hal-00766149

Submitted on 17 Dec 2012

HAL is a multi-disciplinary open access archive for the deposit and dissemination of scientific research documents, whether they are published or not. The documents may come from teaching and research institutions in France or abroad, or from public or private research centers.
L'archive ouverte pluridisciplinaire HAL, est destinée au dépôt et à la diffusion de documents scientifiques de niveau recherche, publiés ou non, émanant des établissements d'enseignement et de recherche français ou étrangers, des laboratoires publics ou privés. 


\title{
Building strategies to ensure language coexistence in presence of bilingualism
}

\author{
C. Bernard, S. Martin* \\ Cemagref - Laboratoire d'Ingénierie des Systèmes Complexes - 24 av. des Landais BP \\ 50085 - 63172 Aubière Cedex - France
}

\begin{abstract}
For twenty years many authors have attempted to model language competition. Some models involve two different languages, others include also a bilingual population. The issues are to understand one language extinction or to determine in which parameter range coexistence is possible. A key parameter is the prestige of one language compare to the other. If this parameter remains constant, coexistence is not sustainable. However, prestige may vary with time. In this article, thanks to the viability theory concepts and tools, we study a set of prestige variations which would allow language coexistence in presence of a bilingual population. Among this set, we emphasise slow viable evolutions with the lowest prestige variations that guarantee coexistence.
\end{abstract}

Keywords: viability domain, slow viable strategies, language coexistence, bilingualism

\section{Introduction}

Many languages might become extinct [1]. It is, therefore, an important challenge to understand language dynamics, and to recognise whether there are measures that can help us preserve some of them. The situation has attracted the interest of many researchers who have analysed language

\footnotetext{
${ }^{*}$ Corresponding author

Email addresses: claire.bernard@cemagref.fr (C. Bernard), sophie.martin@cemagref.fr (S. Martin)
} 
dynamics and developed models of the evolution of the number of their speakers. Among them, Abrams and Strogatz [2] have proposed a mathematical model for studying language competition. The model obtains a good fit to a number of empirical data sets : it satisfactorily fits historical data on the decline of Welsh, Scottish Gaelic, Quechua and other endangered languages, predicts that one of the competing languages will inevitably die out. Actually, the model predicts that whenever two languages compete for speakers, one language will eventually become extinct, the language that dies depending on the initial proportions of speakers of each language and their relative prestige.

Bilingual societies do in fact exist.

In the case of two mutual unintelligible languages, when one language becomes dominant due to political, economical or social advantages, bilingualism may be a transitional stage toward the extinction of the subordinate language [3]. Baggs and Freedman [4] have developed a model for the dynamics of interactions between a bilingual component and a monolingual component of a population. Conditions under which both components of the population will approach a unique and stable steady state were investigated. This two-dimensional model is based on Lotka-Volterra and Holling predator-prey paradigms. Wyburn and Hayward [5] identified four possible scenarios in the long-term future of the bilingual population depending on the model parameter values. El-Owaidy and Ismail [6] have extended the model to describe the dynamics of the interactions of a population with three monolingual components and a component which is trilingual in these three languages and derived criteria for persistence or extinction of these groups. Bilingual societies are thought by Abrams and Strogatz [2] to be, in most cases at least, unstable situations resulting from the recent merging of formerly separate communities with different languages. However, Mira and Paredes [7] have extended the Abrams and Strogatz's work to model bilingualism explicitly, accounting for the fact that some individuals may speak both of the competing languages. They propose a three-dimensional model which variables are the expected aggregate behaviour of the whole population split into three groups : monolingual speakers of the first language, monolingual speakers of the second language and bilingual speakers. They suggest that stable bilingualism may be possible, and that whether it occurs or not may depend on the degree of similarity between the two competing languages. Castello et al. [8] also propose a generalisation of the microscopic version of the Abrams and Strogatz's model for two socially equivalent lan- 
guages, to include the effects of bilingualism. A global consensus state is reached with probability one. Within the assumptions and limitations of their model, their results imply that bilingualism is not an efficient mechanism to stabilise language diversity.

Minett and Wang [9] propose a slightly different model with the same three state variables as Mira and Paredes' one. Guided by Crystal's work [1] on the main mechanisms of intervention by which language coexistence may be attempted, they consider the possibility of an evolution of the two languages relative prestige (the prestige was considered as a constant parameter in the previously cited references). They define several prestige evolution functions and they study their influence on the language coexistence.

The prestige measures the status associated to a language due to individual and social advantages related to the use of that language, being higher according to its presence in education, religion, administration and the media. Modifying the prestige of one language is one of the six main mechanisms of intervention identified by [1]. As Chapel et al. [10] in the absence of bilingualism, we assume that public action can modify the prestige of a language, but that its variation at each time step is bounded. In the context of the explicit modelling of a bilingual group, we aim at determining a set of strategies that allow maintenance of both monolingual groups. This is an inverse problem different from Minett and Wang's problematic [9] that is the direct problem of the determination of the efficiency of predefined strategies.

We adopt a viability theory approach [11]: viability theory provides theoretical concepts and practical tools, to study the compatibility between a control dynamical system and a subset in the state space; especially, a viability domain is defined as a subset of the state space such that an evolution starting from it can be maintained inside it. The determination of a viability domain also provides a regulation map that allows to build strategies to remain inside it; in the context of language maintenance, it provides sets of prestige variations according to the system state that allows coexistence of two monolingual groups.

This paper is organised as follows: first, we introduce the language competition model with two monolingual groups and a bilingual one with a brief stability analysis; then, we describe the constraint set defined by the coexistence criteria and how to build inside it a viability domain thanks to the concept of contingent cone; finally, we derive the associated regulation map, which allows to build strategies ensuring coexistence, the slow viable strategies in particular, that exhibit the lowest prestige variations along the 
evolution.

\section{The model description}

In the Abrams and Strogatz's model [2] and in those inspired from it, the assumption is made that the population size remains constant. And, consequently, the variables are the proportion of different groups of speakers. The population is made of two groups, the monolingual speakers of language $A$ and the monolingual speakers of language $B$, and the model is one-dimensional with $\sigma_{A}$ the proportion of speakers of $A\left(\sigma_{B}=1-\sigma_{A}\right)$. In the model including bilingualism, the population is made of three groups, the monolingual speakers of language $A$, the monolingual speakers of language $B$, and the bilingual speakers $A B$; and the model is two-dimensional with $\sigma_{A}$ the proportion of speakers of $A$ and $\sigma_{B}$ the proportion of speakers of $B$ $\left(\sigma_{A B}=1-\sigma_{A}-\sigma_{B}\right)$.

In any linguistic subpopulation, there are forces and influences which one group exerts on members of the other to switch languages. In the Abrams and Strogatz's model, the rate at which speakers of one language switch to become speakers of the second language depends on the attractiveness of this second language. In their most general conception of attractiveness, Abrams and Strogatz assume that a language has greater attractiveness the more monolingual speakers it has and the greater its prestige is. They state $P_{B \rightarrow A}$, the fraction of group $B$ that transfers to group $A$ per unit time :

$$
P_{B \rightarrow A}=s_{A} \sigma_{A}^{a} \text {. }
$$

$s_{A}$ denotes the prestige of language $A$, and $a$ is a parameter that models how the attractiveness of $A$ scales with the proportion of speakers of $A$. The attractiveness of $B$ to speakers of $A$ can be stated similarly.

The rate of change of $\sigma_{A}$ is given by

$$
\frac{d \sigma_{A}}{d t}=\sigma_{B} P_{B \rightarrow A}-\sigma_{A} P_{A \rightarrow B}
$$

(with analogous equation for $\frac{d \sigma_{B}}{d t}$ ).

Extending the Abrams ans Strogatz's model by explicitly modelling bilingualism, and consequently introducing a third class of speakers, $A B$, who speak both $A$ and $B$, Equation (2) becomes: 


$$
\frac{d \sigma_{A}}{d t}=\sigma_{B} P_{B \rightarrow A}+\sigma_{A B} P_{A B \rightarrow A}-\sigma_{A}\left(P_{A \rightarrow B}+P_{A \rightarrow A B}\right)
$$

(with analogous equations for $\frac{d \sigma_{B}}{d t}$ and $\frac{d \sigma_{A B}}{d t}$ ).

The transitions $A \rightarrow B$ and $B \rightarrow A$ are exceedingly rare in practice [12]. We therefore model only transitions of the four types $A \rightarrow A B, A B \rightarrow A$, $A \rightarrow A B$, and $A B \rightarrow B$ as [8] and [9] $\left(P_{A \rightarrow B}=P_{B \rightarrow A}=0\right)$.

The moving rates depend on the Abrams and Strogatz's definition of language attractiveness (Eq. 1). As [8], we assume an asymmetry between monolinguals and bilinguals: $A \rightarrow A B$ (resp. $B \rightarrow A B$ ) at a rate proportional to the attractiveness of the monolingual speakers of $A$ (resp. $B$ ); $A B \rightarrow A$ (resp. $A B \rightarrow B$ ) at a rate proportional to the attractiveness of the whole speakers of $A$, including the bilingual ones (hence, some bilinguals can become monolingual speakers of $A$ even if $A$ has no monolingual speakers):

$$
\begin{aligned}
P_{A B \rightarrow A} & =\left(1-\sigma_{B}\right)^{a} s_{A} \\
P_{A \rightarrow A B} & =\sigma_{B}^{a} s_{B} .
\end{aligned}
$$

Consequently, the two-dimensional model is defined by

$$
\begin{aligned}
\frac{d \sigma_{A}}{d t} & =\left(1-\sigma_{A}-\sigma_{B}\right)\left(1-\sigma_{B}\right)^{a} s_{A}-\sigma_{A} \sigma_{B}^{a} s_{B} \\
\frac{d \sigma_{B}}{d t} & =\left(1-\sigma_{A}-\sigma_{B}\right)\left(1-\sigma_{A}\right)^{a} s_{B}-\sigma_{B} \sigma_{A}^{a} s_{A} .
\end{aligned}
$$

For convenience, we will assume, that $s_{A}+s_{B}=1$, allowing us to substitute $s_{A}=s$ and $s_{B}=1-s_{A}$.

Remark 1. If the value of the prestige, $s$, is constant in ]0; 1 [, the dynamics (5) has three equilibria: $(0,1)$ and $(1,0)$ which are stable and $\left(\sigma_{A, e}, \sigma_{B, e}\right)$, $\sigma_{A, e}>0, \sigma_{B, e}>0$ which is unstable. Consequently, one language is doomed to become extinct.

In this study, we consider that the prestige, $s$, can evolve (modified by public action for instance), but that its variation at each time step is bounded. We also assume a kind of equivalence between the two languages in the ability of increasing their prestige, so the lower bound of the set of admissible controls, $U$, is the opposite of its upper one:

$$
\begin{aligned}
& \frac{d s}{d t}=u \\
& u \in U:=[-\bar{u} ; \bar{u}], \bar{u}>0 .
\end{aligned}
$$


We propose to find strategies on the prestige variations to maintain a given level of monolingual speakers in both languages, $\underline{\sigma}$, that is to solve the following viability problem :

$$
\left\{\begin{aligned}
\frac{d \sigma_{A}}{d t} & =\left(1-\sigma_{A}-\sigma_{B}\right)\left(1-\sigma_{B}\right)^{a} s-\sigma_{A} \sigma_{B}^{a}(1-s) \\
\frac{d \sigma_{B}}{d t} & =\left(1-\sigma_{A}-\sigma_{B}\right)\left(1-\sigma_{A}\right)^{a}(1-s)-\sigma_{B} \sigma_{A}^{a} s \\
\frac{d s}{d t} & =u \\
u & \in U
\end{aligned}\right.
$$

and

$$
\forall t \geq 0,\left\{\begin{array}{r}
0<\underline{\sigma} \leq \sigma_{A}(t) \leq 1 \\
0<\underline{\sigma} \leq \sigma_{B}(t) \leq 1 \\
0 \leq s(t) \leq 1
\end{array}\right.
$$

Necessarily, $\bar{u}>0$ and $0<\underline{\sigma} \leq 0.5$. In the following sections of this paper, we do not fix a value for $\underline{\sigma}$, but we consider that reasonable values for this parameter are lower than 0.3 .

Remark 2. Let the functions $f_{1}, f_{2}, f$ defined on $\mathbb{R}^{+2} \times \mathbb{R}^{2}$ by :

$$
\begin{aligned}
& f_{1}(x, y, z):=(1-x-y)(1-y)^{a} z-x y^{a}(1-z) \\
& f_{2}(x, y, z):=(1-x-y)(1-x)^{a}(1-z)-x^{a} y z
\end{aligned}
$$

and

$$
f(x, y, z, u):=\left(f_{1}(x, y, z), f_{2}(x, y, z), u\right)
$$

The control system $(U, f)$ defines the dynamics of the model :

$$
\left\{\begin{array}{cl}
\left(\sigma_{A}^{\prime}, \sigma_{B}^{\prime}, s^{\prime}\right) & =f\left(\sigma_{A}, \sigma_{B}, s, u\right) \\
u & \in U .
\end{array}\right.
$$

1. The control system $(U, f)$ is Marchaud $^{1}$.

2. The functions $f_{1}$ and $f_{2}$ have the following symmetry property :

$$
f_{1}(x, y, z)=f_{2}(y, x,(1-z)) \text {. }
$$

\footnotetext{
${ }^{1}$ It satisfies the following conditions :

(i) $\operatorname{Graph}(U)$ is closed

(ii) $\mathrm{f}$ is continuous

(iii) the velocity subsets $F(x):=\{f(x, u)\}_{u \in U(x)}$ are convex

(iv) $f$ and $U$ have linear growth
} 


\section{Building a viability domain}

\subsection{Definition of a viability domain and the viability theorem}

We first recall the definitions of the contingent cone and the viability domain [11]. Let $X$ be a finite dimensional vector space.

Definition 1 (Contingent cone). Let $K$ be a subset of $X$ and $x \in K$, the contingent cone $T_{K}(x)$ to $K$ at $x$ is the closed cone of elements $v$ such that

$$
\lim \inf _{h \rightarrow 0+} \frac{d(x+h v, K)}{h}=0
$$

If $K$ is differentiable at $x$, the contingent cone is the tangent space.

Definition 2 (Viability domain). Let $F: X \rightsquigarrow X$ be a non trivial setvalued map. A subset $K \subset \operatorname{Dom}^{2}(F)$ is a viability domain of $F$ if and only if

$$
\forall x \in K, F(x) \cap T_{K}(x) \neq \emptyset
$$

Aubin [11] also shows the link between viability domains and the existence of viable solutions.

Definition 3 (Viable function). Let $K$ be a subset of $X$. A function $x($. from $[0 ;+\infty[$ is viable in $K$ if $\forall t \geq 0, x(t) \in K$.

Definition 4 (Regulation map). Consider a system $(U, f)$ described by a feedback map $U$ and dynamics $f$. We associate with any subset $K \subset \operatorname{Dom}(U)$ the regulation map $R_{K}: K \rightsquigarrow U$ defined by

$$
\forall x \in K, R_{K}(x):=\left\{u \in U(x) \mid f(x, u) \in T_{K}(x)\right\}
$$

It is worth noting that $K$ is a viability domain if and only if the regulation map $R_{K}$ is strict (has nonempty values).

\footnotetext{
${ }^{2}$ Let $F: X \rightsquigarrow X$ be a non trivial set-valued map,

$$
\operatorname{Dom}(F)=\{x \in X \text { such that } F(x) \neq \emptyset\}
$$
}


Theorem 1 (Viability theorem). Let us consider a Marchaud control system $(U, f)$ and a closed subset $K \subset \operatorname{Dom}(U)$ of $X$. Let $F(x):=\{f(x, u)\}_{u \in U(x)}$. If $K$ is a viability domain under $F$, then for any initial state $x_{0} \in K$, there exists a viable solution on $[0,+\infty[$ to differential inclusion:

$$
\left\{\begin{array}{l}
\text { For almost all } t \geq 0, x^{\prime}(t)=f(x(t), u(t)) \\
\text { where } u(t) \in U(x(t))
\end{array}\right.
$$

Furthermore, any control function regulating a viable solution $x($.$) obeys the$ regulation law

$$
\text { for almost all } t, u(t) \in R_{K}(x(t)) \text {. }
$$

Consequently, if the whole constraint set is a viability domain, whatever the initial state, there exists a control function that governs an evolution which remains in this constraint set. If the whole constraint set is not a viability domain, finding a subset which is a viability domain guarantees the existence of a viable evolution from any starting point inside it.

3.2. Geometric description of the constraint set associated with the coexistence of both monolingual groups

We denote by $K$ the constraint set corresponding to the coexistence of both monolingual groups (Eq. 8). Its boundary is made of 5 faces with $\underline{\sigma} \in] 0 ; 0.3]$ (Fig. 1):

- $F_{0}:\left\{\left(\sigma_{A}, \sigma_{B}, s\right) \in \mathbb{R}^{3} \mid \sigma_{A} \geq \underline{\sigma}, \sigma_{B} \geq \underline{\sigma}, \sigma_{A}+\sigma_{B} \leq 1, s=0\right\}$

- $F_{1}:\left\{\left(\sigma_{A}, \sigma_{B}, s\right) \in \mathbb{R}^{3} \mid \sigma_{A} \geq \underline{\sigma}, \sigma_{B} \geq \underline{\sigma}, \sigma_{A}+\sigma_{B} \leq 1, s=1\right\}$

- $F_{2}:\left\{\left(\sigma_{A}, \sigma_{B}, s\right) \in \mathbb{R}^{3} \mid \sigma_{A} \geq \underline{\sigma}, \sigma_{B} \geq \underline{\sigma}, \sigma_{A}+\sigma_{B}=1,0 \leq s \leq 1\right\}$

- $F_{3}:\left\{\left(\sigma_{A}, \sigma_{B}, s\right) \in \mathbb{R}^{3} \mid \sigma_{A}=\underline{\sigma}, \sigma_{B} \geq \underline{\sigma}, \sigma_{A}+\sigma_{B} \leq 1,0 \leq s \leq 1\right\}$

- $F_{4}:\left\{\left(\sigma_{A}, \sigma_{B}, s\right) \in \mathbb{R}^{3} \mid \sigma_{A} \geq \underline{\sigma}, \sigma_{B}=\underline{\sigma}, \sigma_{A}+\sigma_{B} \leq 1,0 \leq s \leq 1\right\}$

3.3. Intersection between the set-valued map describing the dynamics, $F$, and the contingent cones to the constraint set, $T_{K}$

If $\left(\sigma_{A}, \sigma_{B}, s\right) \in \stackrel{\circ}{K}{ }^{3}, T_{K}\left(\sigma_{A}, \sigma_{B}, s\right)=\mathbb{R}^{3}$ and consequently, $T_{K}\left(\sigma_{A}, \sigma_{B}, s\right) \cap$ $F\left(\sigma_{A}, \sigma_{B}, s\right) \neq \emptyset$.

We then study the intersection on the boundary of $K$.

\footnotetext{
${ }^{3} \stackrel{\circ}{K}:=\left\{x \in K \mid \exists \epsilon>0\right.$ such that $\left.B_{o}(x, \epsilon) \subset K\right\}$ where $B_{o}(x, \epsilon):=\{y \in X \mid\|x-y\|<\epsilon\}$
} 


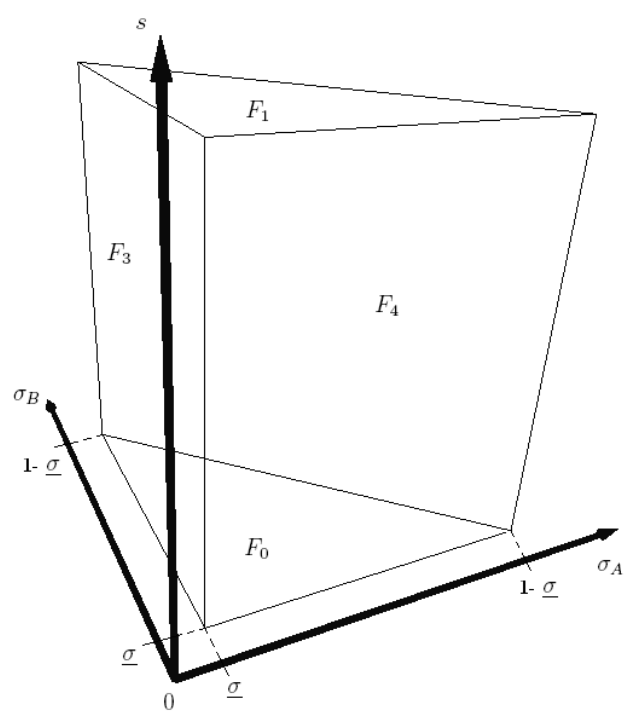

Figure 1: The constraint set $K$

\subsubsection{On faces $F_{0}, F_{1}$ and $F_{2}$}

- If $\left(\sigma_{A}, \sigma_{B}, s\right) \in F_{0}, T_{K}\left(\sigma_{A}, \sigma_{B}, s\right)=\mathbb{R}^{2} \times \mathbb{R}^{+}$, so $\forall u \in[0, \bar{u}] \subset[-\bar{u}, \bar{u}]$, $f\left(\sigma_{A}, \sigma_{B}, s, u\right) \in T_{K}\left(\sigma_{A}, \sigma_{B}, s\right)$.

- If $\left(\sigma_{A}, \sigma_{B}, s\right) \in \stackrel{\circ}{F}_{1}, T_{K}\left(\sigma_{A}, \sigma_{B}, s\right)=\mathbb{R}^{2} \times \mathbb{R}^{-}$, so $\forall u \in[-\bar{u}, 0] \subset[-\bar{u}, \bar{u}]$, $f\left(\sigma_{A}, \sigma_{B}, s, u\right) \in T_{K}\left(\sigma_{A}, \sigma_{B}, s\right)$.

- If $\left(\sigma_{A}, \sigma_{B}, s\right) \in \stackrel{\circ}{F_{2}}, T_{K}\left(\sigma_{A}, \sigma_{B}, s\right)=\left\{(x, y, z) \in \mathbb{R}^{3} \mid x+y \leq 0\right\}$, so $\forall u \in[-\bar{u}, \bar{u}], f\left(\sigma_{A}, \sigma_{B}, s, u\right) \in T_{K}\left(\sigma_{A}, \sigma_{B}, s\right)$.

Then,

- If $\left(\sigma_{A}, \sigma_{B}, s\right) \in F_{0} \cap F_{2}, T_{K}\left(\sigma_{A}, \sigma_{B}, s\right)=\mathbb{R}^{2} \times \mathbb{R}^{+}$, so $\forall u \in[0, \bar{u}] \subset$ $[-\bar{u}, \bar{u}], f\left(\sigma_{A}, \sigma_{B}, s, u\right) \in T_{K}\left(\sigma_{A}, \sigma_{B}, s\right)$.

- If $\forall\left(\sigma_{A}, \sigma_{B}, s\right) \in F_{1} \cap F_{2}, T_{K}\left(\sigma_{A}, \sigma_{B}, s\right)=\mathbb{R}^{2} \times \mathbb{R}^{-}$, so $\forall u \in[-\bar{u}, 0] \subset$ $[-\bar{u}, \bar{u}], f\left(\sigma_{A}, \sigma_{B}, s, u\right) \in T_{K}\left(\sigma_{A}, \sigma_{B}, s\right)$. 


\subsubsection{On faces $F_{3}$ and $F_{4}$}

We study the intersection between $F$ and $T_{K}$ on $F_{3}$ and $F_{4}$. We remind that the dynamics has a symmetry property (Remark. 2). Furthermore, the constraint set is symmetrical by the transformation :

$$
\left(\sigma_{A}, \sigma_{B}, s\right) \rightarrow\left(\sigma_{B}, \sigma_{A}, 1-s\right) .
$$

Consequently the results for $F_{4}$ will be deduced from the ones for $F_{3}$.

If $\left(\sigma_{A}, \sigma_{B}, s\right) \in \stackrel{\circ}{F}_{3}, T_{K}\left(\sigma_{A}, \sigma_{B}, s\right)=\mathbb{R}^{+} \times \mathbb{R}^{2}$.

And $f_{1}\left(\underline{\sigma}, \sigma_{B}, s\right) \geq 0 \Leftrightarrow s \geq \frac{\underline{\sigma} \sigma_{B}^{a}}{\left(1-\underline{\sigma}-\sigma_{B}\right)\left(1-\sigma_{B}\right)^{a}+\underline{\sigma} \sigma_{B}^{a}}:=s_{0}\left(\underline{\sigma}, \sigma_{B}\right)$.

So, if $s<s_{0}\left(\underline{\sigma}, \sigma_{B}\right), T_{K}\left(\underline{\sigma}, \sigma_{B}, s\right) \cap F\left(\underline{\sigma}, \sigma_{B}, s\right)=\emptyset$.

So $K$ is not a viability domain. Figure 2 summarizes this study on the intersection between the contingent cones to the constraint set and the dynamics.

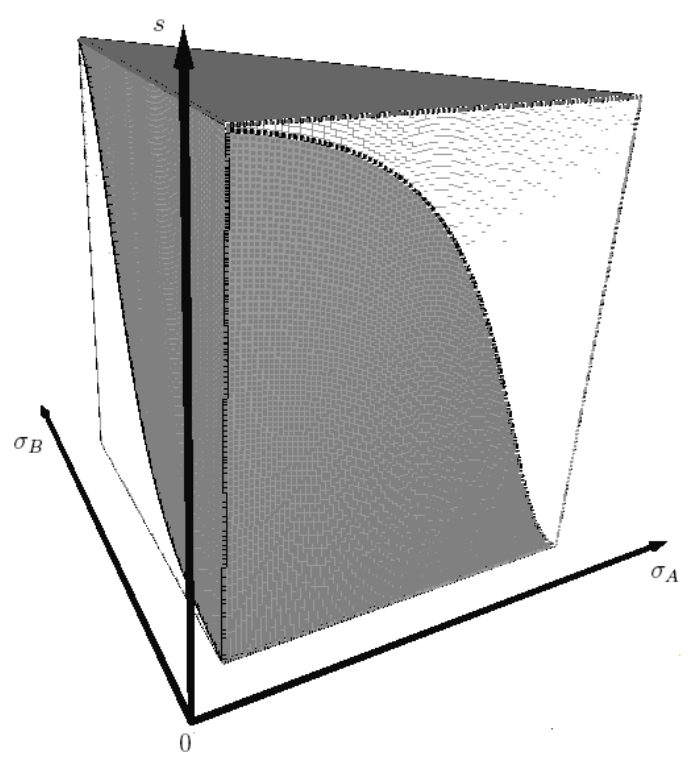

Figure 2: The area where the intersection between the contingent cone to the constraint set and the dynamics is not empty is colored grey. The area where it is empty is white dashed.

\subsection{Sculpting the constraint set}

On face $F_{3}, \sigma_{A}=\underline{\sigma}$, and when $s=s_{0}\left(\underline{\sigma}, \sigma_{B}\right)$,

$\sigma_{A}^{\prime}\left(\underline{\sigma}, \sigma_{B}, s_{0}\left(\underline{\sigma}, \sigma_{B}\right)\right)=f_{1}\left(\underline{\sigma}, \sigma_{B}, s_{0}\left(\underline{\sigma}, \sigma_{B}\right)\right)=0$. Then to go on analysing the 
possible constraint violation, we have to study the sign of $\sigma_{A}^{\prime \prime}$.

$$
\frac{\partial^{2} \sigma_{A}}{\partial t^{2}}\left(\sigma_{A}, \sigma_{B}, s\right)=d f_{1}\left(\sigma_{A}, \sigma_{B}, s\right) \cdot\left(f_{1}\left(\sigma_{A}, \sigma_{B}, s\right), f_{2}\left(\sigma_{A}, \sigma_{B}, s\right), u\right)
$$

From (Eq. 9), $d f_{1}\left(\sigma_{A}, \sigma_{B}, s\right)=d\left(\left(1-\sigma_{A}-\sigma_{B}\right)\left(1-\sigma_{B}\right)^{a} s-\sigma_{A} \sigma_{B}^{a}(1-s)\right)$ and

$$
\begin{aligned}
& \frac{\partial f_{1}}{\partial \sigma_{A}}\left(\sigma_{A}, \sigma_{B}, s\right)=-\left(\left(1-\sigma_{B}\right)^{a} s+(1-s) \sigma_{B}{ }^{a}\right) \\
& \frac{\partial f_{1}}{\partial \sigma_{B}}\left(\sigma_{A}, \sigma_{B}, s\right)=-\left(\left(1-\sigma_{B}\right)^{a} s+a(1-s) \sigma_{A} \sigma_{B}^{a-1}+a\left(1-\sigma_{A}-\sigma_{B}\right)\left(1-\sigma_{B}\right)^{a-1}\right) \\
& \frac{\partial f_{1}}{\partial s}\left(\sigma_{A}, \sigma_{B}, s\right)=\left(1-\sigma_{A}-\sigma_{B}\right)\left(1-\sigma_{B}\right)^{a}+\sigma_{A} \sigma_{B}^{a}
\end{aligned}
$$

Then

$$
\begin{aligned}
\frac{\partial^{2} \sigma_{A}}{\partial t^{2}}=- & \left(\left(1-\sigma_{B}\right)^{a} s+(1-s) \sigma_{B}{ }^{a}\right) f_{1}\left(\sigma_{A}, \sigma_{B}, s\right) \\
- & \left(\left(1-\sigma_{B}\right)^{a} s+a(1-s) \sigma_{A} \sigma_{B}^{a-1}+a\left(1-\sigma_{A}-\sigma_{B}\right)\left(1-\sigma_{B}\right)^{a-1} s\right) \\
& f_{2}\left(\sigma_{A}, \sigma_{B}, s\right) \\
+ & \left(\left(1-\sigma_{A}-\sigma_{B}\right)\left(1-\sigma_{B}\right)^{a}+\sigma_{A} \sigma_{B}^{a}\right) u
\end{aligned}
$$

So $\frac{\partial^{2} \sigma_{A}}{\partial t^{2}}\left(\underline{\sigma}, \sigma_{B}, s=s_{0}\left(\underline{\sigma}, \sigma_{B}\right)\right) \geq 0$, when :

$$
\begin{aligned}
-\left(\left(1-\sigma_{B}\right)^{a} s+a(1-s) \underline{\sigma} \sigma_{B}^{a-1}+a\left(1-\underline{\sigma}-\sigma_{B}\right)\left(1-\sigma_{B}\right)^{a-1} s\right) & f_{2}\left(\underline{\sigma}, \sigma_{B}, s\right) \\
+\left(\left(1-\underline{\sigma}-\sigma_{B}\right)\left(1-\sigma_{B}\right)^{a}+\underline{\sigma} \sigma_{B}^{a}\right) & u \\
& \geq 0
\end{aligned}
$$

That is, since $u$ multiplicative factor is strictly positive on $F_{3}\left(\sigma_{B} \geq \underline{\sigma}>\right.$ 0):

$$
\begin{aligned}
u & \geq \frac{\left(1-\sigma_{B}\right)^{a} s+a\left(1-\underline{\sigma}-\sigma_{B}\right)\left(1-\sigma_{B}\right)^{a-1} s+a(1-s) \underline{\sigma} \sigma_{B}^{a-1}}{\left(1-\underline{\sigma}-\sigma_{B}\right)\left(1-\sigma_{B}\right)^{a}+\underline{\sigma} \sigma_{B}^{a}} f_{2}\left(\underline{\sigma}, \sigma_{B}, s\right) \\
& =\frac{\partial s_{0}}{\partial \sigma_{B}}\left(\underline{\sigma}, \sigma_{B}\right) f_{2}\left(\underline{\sigma}, \sigma_{B}, s\right)
\end{aligned}
$$

As $u \in U=[-\bar{u} ; \bar{u}]$, a necessary and sufficient condition for the existence of a control such that $\frac{\partial^{2} \sigma_{A}}{\partial t^{2}}\left(\underline{\sigma}, \sigma_{B}, s=s_{0}\left(\underline{\sigma}, \sigma_{B}\right)\right)$ is greater or equal to 0 is that:

$$
c\left(\underline{\sigma}, \sigma_{B}\right):=\frac{\partial s_{0}}{\partial \sigma_{B}}\left(\underline{\sigma}, \sigma_{B}\right) f_{2}\left(\underline{\sigma}, \sigma_{B}, s\right) \leq \bar{u}
$$

Figure 3 displays the plot of function $c: \sigma_{B} \rightarrow c\left(\underline{\sigma}, \sigma_{B}\right)$ for different values of $\underline{\sigma} \in] 0 ; 0.3]$.

We first note that $c\left(\underline{\sigma}, \sigma_{B}\right)$ and $f_{2}\left(\underline{\sigma}, \sigma_{B}, s_{0}\left(\underline{\sigma}, \sigma_{B}\right)\right)$ have the same sign since $\frac{\partial s_{0}}{\partial \sigma_{B}}\left(\underline{\sigma}, \sigma_{B}\right) \geq 0$ (Eq. 22).

And $f_{2}\left(\underline{\sigma}, \sigma_{B}, s_{0}\left(\underline{\sigma}, \sigma_{B}\right)\right) \leq 0$ when $s_{0}\left(\underline{\sigma}, \sigma_{B}\right) \geq s_{1}\left(\underline{\sigma}, \sigma_{B}\right):=\frac{\left(1-\underline{\sigma}-\sigma_{B}\right)(1-\underline{\sigma})^{a}}{\left(1-\underline{\sigma}-\sigma_{B}\right)(1-\underline{\sigma})^{a}+\sigma_{B} \underline{\sigma}^{a}}$. 


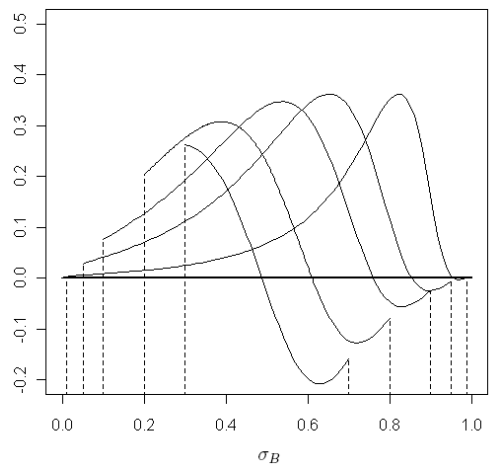

(a)

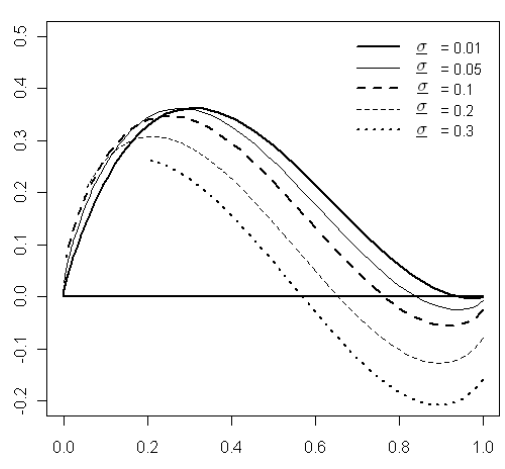

(b)

Figure 3: Plot of function $c(\underline{\sigma},):$. (a) $\sigma_{B} \in[\underline{\sigma} ; 1-\underline{\sigma}] \rightarrow c\left(\underline{\sigma}, \sigma_{B}\right)$, (b) $s \in\left[s_{0}(\underline{\sigma}, \underline{\sigma}) ; 1\right] \rightarrow$ $c(\underline{\sigma}, s)$ for different values of $\underline{\sigma} \in] 0 ; 0.3]$.

Let $\bar{\sigma}(\underline{\sigma})$ such that $s_{0}(\underline{\sigma}, \bar{\sigma}(\underline{\sigma}))=s_{1}(\underline{\sigma}, \bar{\sigma}(\underline{\sigma})), \bar{\sigma}(\underline{\sigma})<1-\underline{\sigma}$ and for all $\sigma_{B} \in[\bar{\sigma}(\underline{\sigma}) ; 1-\underline{\sigma}], f_{2}\left(\underline{\sigma}, \sigma_{B}, s_{0}\left(\underline{\sigma}, \sigma_{B}\right)\right) \leq 0$ and $c\left(\underline{\sigma}, \sigma_{B}\right) \leq 0$.

Moreover, let $\hat{\sigma}(\underline{\sigma}, \bar{u})=\min \left\{\sigma_{B} \in[\underline{\sigma}, \bar{\sigma}] \mid \forall \sigma \geq \sigma_{B} c(\underline{\sigma}, \sigma) \leq \bar{u}\right\}$. Necessarily, $\hat{\sigma}<\bar{\sigma}<1-\underline{\sigma}$ since $\bar{u}>0$.

Three situations may occur depending on the values of $\underline{\sigma}$ and $\bar{u}$ as illustrated in Figure 4.

- Case $1: \hat{\sigma}=\underline{\sigma}$ (Figure 4(a))

- Case $2: \hat{\sigma}>\underline{\sigma}$ and for all $\underline{\sigma} \leq \sigma_{B}<\hat{\sigma}, c\left(\underline{\sigma}, \sigma_{B}\right)>\bar{u}$ (Figure 4(b))

- Case 3: $\hat{\sigma}>\underline{\sigma}$ and there exists $\tilde{\sigma}$ such that $\underline{\sigma} \leq \tilde{\sigma}<\hat{\sigma}$ and for all $\sigma_{B} \mid \underline{\sigma} \leq \sigma_{B} \leq \tilde{\sigma}, c\left(\underline{\sigma}, \sigma_{B}\right) \leq \bar{u}($ Figure $4(\mathrm{c})$ )

Depending on case 1, 2 or 3 , the building procedure of the viability domain is slightly different :

\subsubsection{Case $1: \hat{\sigma}=\underline{\sigma}$}

Let $\Phi(\sigma, t):[\underline{\sigma} ; 1-\underline{\sigma}] \times\left[0 ;+\infty\left[\rightarrow \mathbb{R}^{3}\right.\right.$ such that

$$
\left\{\begin{array}{l}
\Phi(\sigma, 0)=\left(\underline{\sigma}, \sigma, s_{0}(\underline{\sigma}, \sigma)\right) \\
\frac{\partial \Phi(\sigma, t)}{\partial t}=\left(-f_{1}(\Phi(\sigma, t)),-f_{2}(\Phi(\sigma, t)),-\bar{u}\right) .
\end{array}\right.
$$

We are interested in the intersection between $\{\Phi(\sigma, t) \mid(\sigma, t) \in[\underline{\sigma} ; 1-\underline{\sigma}] \times$ $[0 ;+\infty[\}$ and the constraint set $K$. 


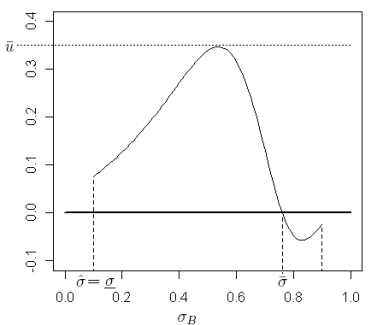

(a)

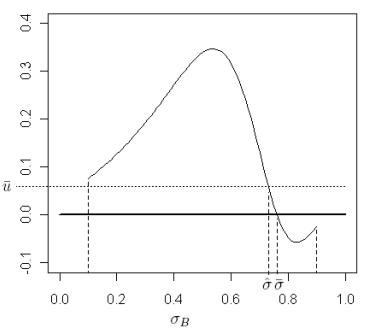

(b)

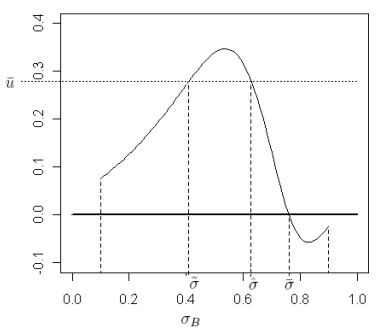

(c)

Figure 4: Plot of $c(\underline{\sigma},$.$) for \underline{\sigma}=0.1$. Three situations can occur depending on the value of $\bar{u}$.

We first note that $\forall \sigma \in[\underline{\sigma} ; 1-\underline{\sigma}], \Phi(\sigma, 0) \in K$. Then, $\forall \sigma \in[\underline{\sigma} ; 1-\underline{\sigma}]$, $\exists T \in[0 ; 1 / \bar{u}]$ such that $\Phi(\sigma, T) \notin K$ since $K \subset \mathbb{R} \times \mathbb{R} \times \mathbb{R}^{+}$and the projection of $\Phi(\sigma, t)$ on the $s$-coordinate decreases from $s_{0}(\sigma)$ with constant speed $-\bar{u}$ (Eq.23).

We denote $T(\sigma)=\max \{T \mid \forall t \in[0 ; T], \Phi(\sigma, t) \in K\} . \forall \sigma \in[\underline{\sigma} ; 1-\underline{\sigma}]$, $T(\sigma) \leq 1 / \bar{u}$.

Lemma 1. $T(\underline{\sigma})=0$ and $\Phi(\underline{\sigma}, T(\underline{\sigma})) \in F_{3} \cap F_{4}$ $T(1-\underline{\sigma})=0$ and $\Phi(1-\underline{\sigma}, T(1-\underline{\sigma})) \in F_{3} \cap F_{2}$.

Proof. $\Phi(\underline{\sigma}, 0)=\left(\underline{\sigma}, \underline{\sigma}, s_{0}(\underline{\sigma}, \underline{\sigma})\right) \in F_{3} \cap F_{4}$ and

$$
\begin{aligned}
-f_{2}(\Phi(\underline{\sigma}, 0)) & =-f_{2}\left(\underline{\sigma}, \underline{\sigma}, s_{0}(\underline{\sigma}, \underline{\sigma})\right) & & \\
& =-f_{1}\left(\underline{\sigma}, \underline{\sigma}, 1-s_{0}(\underline{\sigma}, \underline{\sigma})\right) & & \text { (symmetry property) } \\
& <-f_{1}\left(\underline{\sigma}, \underline{\sigma}, s_{0}(\underline{\sigma}, \underline{\sigma})\right) & & \left(f_{1} \text { increases with } s\right. \text { and } \\
& <0 & & \left.s_{0}(\underline{\sigma}, \underline{\sigma})<0.5 \text { when } \underline{\sigma}<0.3\right)
\end{aligned}
$$

So $T(\underline{\sigma})=0$ and $\Phi(\underline{\sigma}, T(\underline{\sigma})) \in F_{3} \cap F_{4}$.

$\Phi(1-\underline{\sigma}, 0)=(\underline{\sigma}, 1-\underline{\sigma}, 1) \in F_{3} \cap F_{2}$ and $-f_{1}(\underline{\sigma}, 1-\underline{\sigma}, 1)-f_{2}(\underline{\sigma}, 1-\underline{\sigma}, 1)>0$, so $T(1-\underline{\sigma})=0$ and $\Phi(1-\underline{\sigma}, T(1-\underline{\sigma})) \in F_{3} \cap F_{2}$.

Lemma 2. Let $\sigma_{0} \in[\underline{\sigma}, 1-\underline{\sigma}]$. If $\left\{\Phi\left(\sigma_{0}, t\right) \mid t \in\left[0, T\left(\sigma_{0}\right)\right]\right\} \cap \partial K=$ $\left\{\Phi\left(\sigma_{0}, 0\right)\right\} \cup\left\{\Phi\left(\sigma_{0}, T\left(\sigma_{0}\right)\right)\right\}$, then $\sigma \rightarrow \Phi(\sigma, T(\sigma))$ is continuous at $\sigma_{0}$.

Proof. By the definition of $T\left(\sigma_{0}\right)$ and since $\Phi$ is continuous, there exists $\bar{\epsilon}>0$ such that $\forall \delta$ such that $0 \leq \epsilon<\bar{\epsilon}, \Phi\left(\sigma_{0}, T\left(\sigma_{0}\right)+\epsilon\right) \notin K$. 
Let dist be the Euclidean distance in $\mathbb{R}^{3}$.

Let $d=\operatorname{dist}\left(K, \Phi\left(\sigma_{0}, T\left(\sigma_{0}\right)+\epsilon\right)\right)>0$ ( $\mathrm{K}$ is a closed subset of $\left.X\right)$.

As $\frac{\partial \phi}{\partial t}(\sigma, t)$ is bounded on any compact subset of $[\underline{\sigma}, 1-\underline{\sigma}] \times\left[0 ;+\infty\left[, \exists \delta_{\sigma}>0\right.\right.$ such that $\forall \delta|| \delta \mid \leq \delta_{\sigma}\left\|\Phi\left(\sigma_{0}+\delta, T\left(\sigma_{0}\right)+\epsilon\right)-\Phi\left(\sigma_{0}, T\left(\sigma_{0}\right)+\epsilon\right)\right\| \leq d / 2$. Therefore, $\Phi\left(\sigma_{0}+\delta, T\left(\sigma_{0}\right)+\epsilon\right) \notin K$ and $T\left(\sigma_{0}+\delta\right) \leq T\left(\sigma_{0}\right)+\epsilon$.

If $\sigma_{0}=\underline{\sigma}$ or $\sigma_{0}=1-\underline{\sigma}, T\left(\sigma_{0}\right)=0$, and $\forall 0<\epsilon<\bar{\epsilon}, \exists \delta_{\sigma}>0$ such that $|\delta|<\delta_{\sigma}$ implies $T\left(\sigma_{0}+\delta\right) \leq \epsilon$. So $T$ is continuous at $\sigma_{0}$.

If $\left.\sigma_{0} \in\right] \underline{\sigma} ; 1-\underline{\sigma}\left[, \frac{\partial \Phi}{\partial t}\left(\sigma_{0}, 0\right)=0\right.$ and $\frac{\partial^{2} \Phi}{\partial t^{2}}\left(\sigma_{0}, 0\right)=\alpha>0$. So $T\left(\sigma_{0}\right)>0$. Moreover, since $\frac{\partial^{2} \Phi}{\partial t^{2}}$ is continuous, $\exists \tilde{\epsilon}>0, \exists \tilde{\delta}_{\sigma}>0$ such that $\forall \delta|| \delta \mid \leq \tilde{\delta}_{\sigma}$, $\forall \epsilon \mid 0 \leq \epsilon \leq \tilde{\epsilon}, \frac{\partial^{2} \Phi}{\partial t^{2}}\left(\sigma_{0}+\delta, \epsilon\right)>\frac{\alpha}{2}$ and $\Phi\left(\sigma_{0}+\delta, \epsilon\right) \in \stackrel{\circ}{K}$.

Let $\tilde{d}=\min _{t \in\left[\tilde{\epsilon} ; T\left(\sigma_{0}\right)-\epsilon\right]} \operatorname{dist}\left(K, \Phi\left(\sigma_{0}, t\right)\right), \tilde{d}>0$ with the assumption of the lemma $\left\{\Phi\left(\sigma_{0}, t\right) \mid t \in\left[0, T\left(\sigma_{0}\right)\right]\right\} \cap \partial K=\left\{\Phi\left(\sigma_{0}, 0\right)\right\} \cup\left\{\Phi\left(\sigma_{0}, T\left(\sigma_{0}\right)\right)\right\}$. So $\underset{\sim}{\exists} \overline{\delta_{\sigma}}>0$, such that $\forall t \in\left[\tilde{\epsilon} ; T\left(\sigma_{0}\right)-\epsilon\right], \forall \delta|| \delta \mid \leq \bar{\delta}_{\sigma},\left\|\Phi\left(\sigma_{0}+\delta, t\right)-\Phi\left(\sigma_{0}, t\right)\right\| \leq$ $\tilde{d} / 2$.

So, $\forall \delta|| \delta \mid \leq \min \left(\tilde{\delta}_{\sigma}, \bar{\delta}_{\sigma}\right), T\left(\sigma_{0}+\delta\right) \geq T\left(\sigma_{0}\right)-\epsilon$.

Finally, $T$ is continuous at $\sigma_{0}$. As $\Phi$ is continuous on $[\underline{\sigma} ; 1-\underline{\sigma}] \times \mathbb{R}^{+}$, so $\sigma \rightarrow \Phi(\sigma, T(\sigma))$ is also continuous at $\sigma_{0}$.

Figure 5 displays the plot of $T(\sigma)$ for the pair $\underline{\sigma}=0.2$ and $\bar{u}=0.32$ belonging to case 1 .

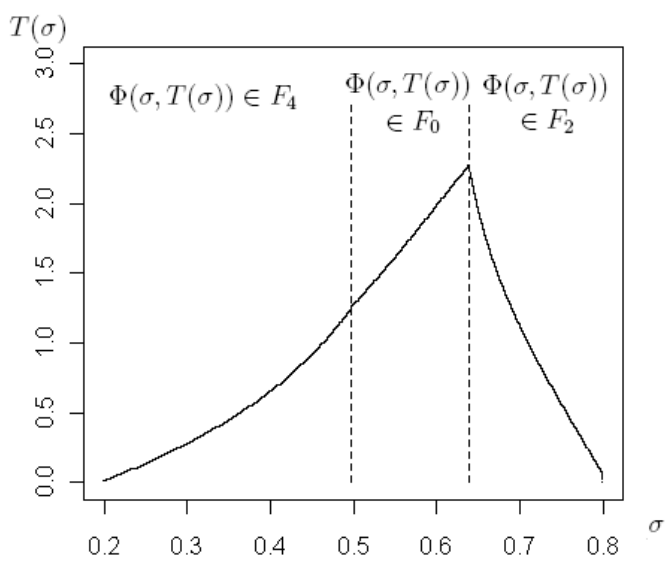

Figure 5: $\underline{\sigma}=0.2$ and $\bar{u}=0.32$. Plot of the function $T:[\underline{\sigma} ; 1-\underline{\sigma}] \rightarrow \mathbb{R}^{+}$

Figure 5 also describes the face $\Phi(\sigma, T(\sigma))$ belongs to : 
- there exists $\underline{\sigma} \leq \sigma_{1}$, such that for all $\sigma \in\left[\underline{\sigma} ; \sigma_{1}\right], \Phi(\sigma, T(\sigma)) \in F_{4}$

- there exists $\sigma_{1} \leq \sigma_{2}$, such that for all $\sigma \in\left[\sigma_{1} ; \sigma_{2}\right], \Phi(\sigma, T(\sigma)) \in F_{0}$

- and for all $\sigma \in\left[\sigma_{2} ; 1-\underline{\sigma}\right], \Phi(\sigma, T(\sigma)) \in F_{2}$.

Corollary 1. $\sigma \rightarrow \Phi(\sigma, T(\sigma))$ is continuous on $[\underline{\sigma}, 1-\underline{\sigma}]$

Proof. Suppose $\Phi(\sigma, T(\sigma))$ is not continuous at $\sigma_{0}$.

From lemma $2, \lim _{\sigma \rightarrow \sigma_{0}^{-}} \Phi(\sigma, T(\sigma))$ (or $\left.\lim _{\sigma \rightarrow \sigma_{0}^{+}} \Phi(\sigma, T(\sigma))\right)$ equals $\Phi\left(\sigma_{0}, T_{1}\right) \neq$ $\Phi\left(\sigma_{0}, T\left(\sigma_{0}\right)\right)$ with $\Phi\left(\sigma_{0}, T_{1}\right) \in \partial K$ and $T_{1}<T\left(\sigma_{0}\right)$.

So $\left(-f_{1}\left(\Phi\left(\sigma_{0}, T_{1}\right)\right),-f_{2}\left(\Phi\left(\sigma_{0}, T_{1}\right)\right),-\bar{u}\right)$ belongs to the contingent cone of $K$ at $\Phi\left(\sigma_{0}, T_{1}\right)$.

Consequently, $\Phi\left(\sigma_{0}, T_{1}\right) \notin F_{0}$ and $\Phi\left(\sigma_{0}, T_{1}\right) \notin F_{2}$ (see subsection 3.3.1).

Moreover, $\Phi\left(\sigma_{0}, T_{1}\right) \notin F_{4}$ if $(\mathrm{H} 1) \bar{u}>\left|\min _{\sigma \in[\underline{\sigma} ; 1-\underline{\sigma}]} c(\underline{\sigma}, \sigma)\right|$.

Actually, if $\Phi\left(\sigma_{0}, T_{1}\right) \in F_{4}, f_{2}\left(\Phi\left(\sigma_{0}\right), T_{1}\right)=0$, and by the symmetry property, $\exists \sigma$ such that $\Phi\left(\sigma_{0}, T_{1}\right)=\left(\sigma, \underline{\sigma}, 1-s_{0}(\underline{\sigma}, \sigma)\right)$. Moreover, let $\Phi_{\sigma_{B}}$ be the projection of $\Phi$ on the $\sigma_{B}$-coordinate, $\frac{\partial^{2} \Phi_{\sigma_{B}}}{\partial t^{2}}\left(\sigma_{0}, T_{1}\right) \geq 0$ implies, using the symmetry property and following the same development as Eq. 20, Eq. 21 and Eq. 22, that $-\bar{u} \geq c(\underline{\sigma}, \sigma)$ which contradicts the assumption (H1).

As $\max _{\sigma \in[\underline{\sigma} ; 1-\underline{\sigma}]} c(\underline{\sigma}, \sigma)>\left|\min _{\sigma \in[\underline{\sigma} ; 1-\underline{\sigma}]} c(\underline{\sigma}, \sigma)\right|$ (Fig. 4(a)), assumption (H1) is satisfied in case 1 .

Figure 6 displays a 3D plot of the intersection between $K$ and the surface $\{\Phi(\sigma, t) \mid(\sigma, t) \in[\underline{\sigma}, 1-\underline{\sigma}] \times[0, T(\sigma)]\}$.

3.4.2. Case 2 : $\hat{\sigma}>\underline{\sigma}$ and (H2) for all $\underline{\sigma} \leq \sigma_{B}<\hat{\sigma}, c\left(\underline{\sigma}, \sigma_{B}\right)>\bar{u}$

Let $\Phi(\sigma, t):[\hat{\sigma} ; 1-\underline{\sigma}] \times[0 ;+\infty] \rightarrow \mathbb{R}^{3}$ defined as in Eq. (23).

As in case $1, T(\sigma):=\max \{T \mid \forall t \in[0 ; T] \Phi(\sigma, t) \in K\}$ and $\forall \sigma \in[\hat{\sigma} ; 1-\underline{\sigma}]$, $T(\sigma) \leq 1 / \bar{u}$.

Lemma 3. $T(\hat{\sigma})=0$ and $\Phi(\hat{\sigma}, T(\hat{\sigma})) \in \stackrel{\circ}{F}_{3}$

$T(1-\underline{\sigma})=0$ and $\Phi(1-\underline{\sigma}, T(1-\underline{\sigma})) \in F_{3} \cap F_{2}$.

Proof. By the definition of $\hat{\sigma}, f_{1}\left(\underline{\sigma}, \hat{\sigma}, s_{0}(\underline{\sigma}, \hat{\sigma})\right)=0$.

Moreover, $d f_{1}\left(\underline{\sigma}, \hat{\sigma}, s_{0}(\underline{\sigma}, \hat{\sigma})\right) . f\left(\underline{\sigma}, \hat{\sigma}, s_{0}(\underline{\sigma}, \hat{\sigma}), \bar{u}\right)=0$.

And let $h\left(\sigma_{A}, \sigma_{B}, s\right):=d f_{1}\left(\sigma_{A}, \sigma_{B}, s\right) . f\left(\left(\sigma_{A}, \sigma_{B}, s, \bar{u}\right)\right.$,

$$
d h\left(\underline{\sigma}, \hat{\sigma}, s_{0}(\underline{\sigma}, \hat{\sigma})\right)\left(0,1, \frac{\partial s_{0}}{\partial \sigma}(\underline{\sigma}, \hat{\sigma})\right)<0 .
$$




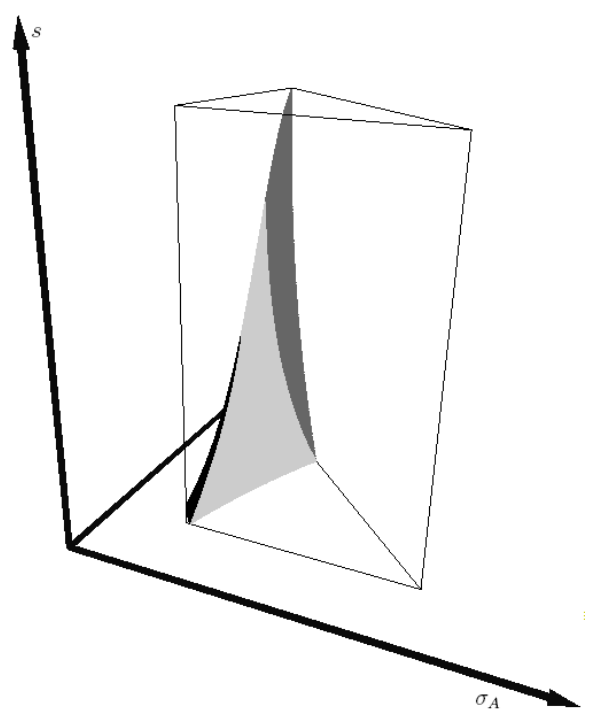

(a)

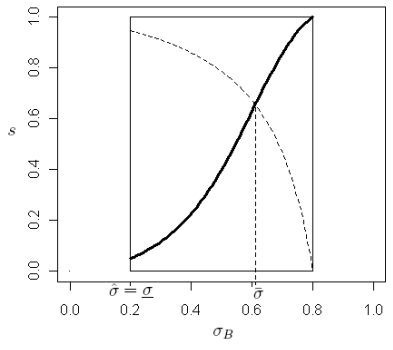

(b)

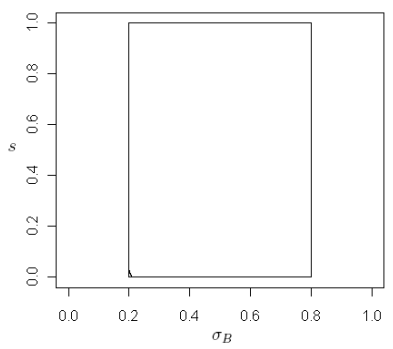

(c)

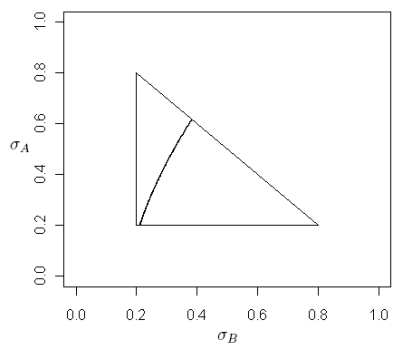

(d)

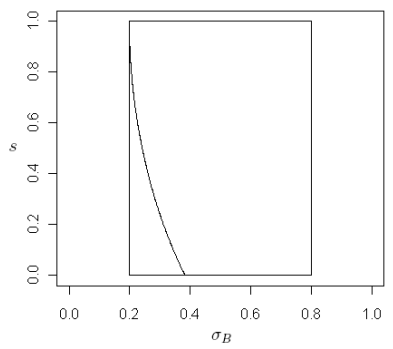

(e)

Figure 6: 6(a) The Euler approximation of $\{\Phi(\sigma, t) \mid \sigma \in[\underline{\sigma}, 1-\underline{\sigma}] \times[0, T(\sigma)]\}$ for $\underline{\sigma}=0.2$ and $\bar{u}=0.32$. The area $\left\{\Phi(\sigma, t) \mid \Phi(\sigma, T(\sigma)) \in F_{4}\right\}$ is colored black. The area $\left\{\Phi(\sigma, t) \mid \Phi(\sigma, T(\sigma)) \in F_{0}\right\}$ is colored light gray. The area $\left\{\Phi(\sigma, t) \mid \Phi(\sigma, T(\sigma)) \in F_{2}\right\}$ is colored dark gray. $6(\mathrm{~b}), 6(\mathrm{c}), 6(\mathrm{~d}), 6(\mathrm{e})$, the intersection between $\Phi(\sigma, t)$ and $F_{3}, F_{4}, F_{0}$ and $F_{2}$.

Let $\Phi_{\sigma_{A}}$ be the $\sigma_{A}$-component of $\Phi, \frac{\partial \Phi_{\sigma_{A}}}{\partial t}(\hat{\sigma}, 0)=-f_{1}\left(\underline{\sigma}, \hat{\sigma}, s_{0}(\underline{\sigma}, \hat{\sigma})\right)=0$. $\frac{\partial^{2} \Phi_{\sigma_{A}}}{\partial t^{2}}(\hat{\sigma}, 0)=-d f_{1}\left(\underline{\sigma}, \hat{\sigma}, s_{0}(\underline{\sigma}, \hat{\sigma})\right) \cdot f\left(\underline{\sigma}, \hat{\sigma}, s_{0}(\underline{\sigma}, \hat{\sigma}), \bar{u}\right)=0$. 
Furthermore,

$$
\begin{aligned}
\frac{\partial^{3} \Phi_{\sigma_{A}}(\hat{\sigma}, 0)}{\partial t^{3}} & =d h\left(\underline{\sigma}, \hat{\sigma}, s_{0}(\underline{\sigma}, \hat{\sigma})\right)\left(-f_{1}\left(\underline{\sigma}, \hat{\sigma}, s_{0}(\underline{\sigma}, \hat{\sigma})\right),-f_{2}\left(\underline{\sigma}, \hat{\sigma}, s_{0}(\underline{\sigma}, \hat{\sigma})\right),-\bar{u}\right) \\
& =d h\left(\underline{\sigma}, \hat{\sigma}, s_{0}(\underline{\sigma}, \hat{\sigma})\right)\left(0,-f_{2}\left(\underline{\sigma}, \hat{\sigma}, s_{0}(\underline{\sigma}, \hat{\sigma})\right),-\bar{u}\right)
\end{aligned}
$$

But, $\left(0,-f_{2}\left(\underline{\sigma}, \hat{\sigma}, s_{0}(\underline{\sigma}, \hat{\sigma})\right),-\bar{u}\right)$ and $\left(0,-1,-\frac{\partial s_{0}}{\partial \sigma}(\underline{\sigma}, \hat{\sigma})\right)$ are collinear with the same direction since $c(\underline{\sigma}, \hat{\sigma})=\bar{u}$, so there exists $\lambda>0$ such that :

$$
\begin{aligned}
d h\left(\underline{\sigma}, \hat{\sigma}, s_{0}(\underline{\sigma}, \hat{\sigma})\right)\left(0,-f_{2}\left(\underline{\sigma}, \hat{\sigma}, s_{0}(\underline{\sigma}, \hat{\sigma})\right),-\bar{u}\right) & =\lambda d h\left(\underline{\sigma}, \hat{\sigma}, s_{0}(\underline{\sigma}, \hat{\sigma})\right)\left(0,-1,-\frac{\partial s_{0}}{\partial \sigma}(\underline{\sigma}, \hat{\sigma})\right) \\
& <0
\end{aligned}
$$

So $\frac{\partial^{3} \Phi_{\sigma_{A}}(\hat{\sigma}, 0)}{\partial t^{3}}<0, T(\hat{\sigma})=0$ and $\Phi(\hat{\sigma}, T(\hat{\sigma})) \in \stackrel{\circ}{F}_{3}$.

Figure 7 displays the plot of $T(\sigma)$ for the pair $\underline{\sigma}=0.1$ and $\bar{u}=0.006$ belonging to case 2 .

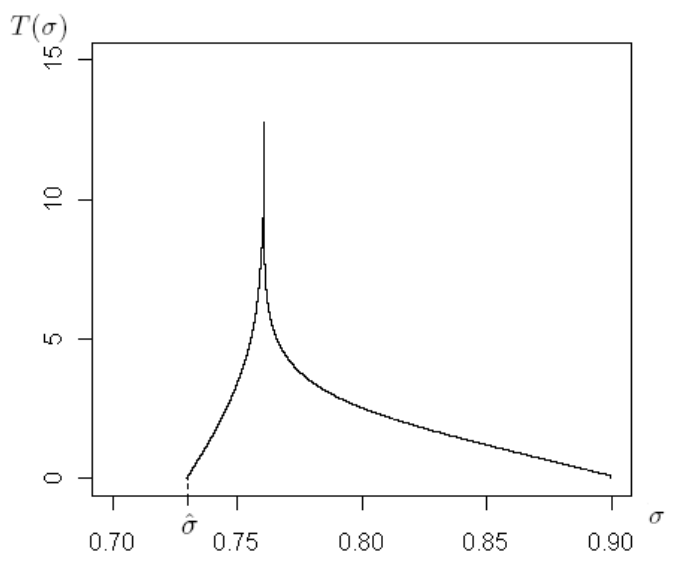

Figure 7: $\underline{\sigma}=0.1$ and $\bar{u}=0.06 . \hat{\sigma} \approx 0.73$. Plot of the function $T:[\hat{\sigma} ; 1-\underline{\sigma}] \rightarrow \mathbb{R}^{+}$

We can not represent on figure 7 the face $\Phi(\sigma, T(\sigma))$ belongs to since some bounds are very close but :

- there exists $\underline{\sigma} \leq \sigma_{1}$, such that for all $\sigma \in\left[\underline{\sigma} ; \sigma_{1}\right], \Phi(\sigma, T(\sigma)) \in F_{3}$

- there exists $\sigma_{1} \leq \sigma_{2}$, such that for all $\sigma \in\left[\sigma_{1} ; \sigma_{2}\right], \Phi(\sigma, T(\sigma)) \in F_{4}$

- there exists $\sigma_{2} \leq \sigma_{3}$, such that for all $\sigma \in\left[\sigma_{2} ; \sigma_{3}\right], \Phi(\sigma, T(\sigma)) \in F_{0}$ 
- and for all $\sigma \in\left[\sigma_{3} ; 1-\underline{\sigma}\right], \Phi(\sigma, T(\sigma)) \in F_{2}$.

Moreover,

Corollary 2. If (H1) is satisfied, $\sigma \rightarrow \Phi(\sigma, T(\sigma))$ is continuous on $[\hat{\sigma}, 1-\underline{\sigma}]$.

Proof. We use the same notations as corrolary 1.

From lemma 2 and lemma $3, \Phi(\sigma, T(\sigma))$ is continuous at $\hat{\sigma}$ and $1-\underline{\sigma}$.

From corollary 1 , if (H1) and if $\Phi(\sigma, T(\sigma))$ is not continuous at $\sigma_{0}, \Phi\left(\sigma_{0}, T_{1}\right) \notin$ $F_{0} \cup F_{2} \cup F_{4}$.

If $\Phi\left(\sigma_{0}, T_{1}\right) \in F_{3}$, since $\Phi\left(\sigma_{0}, 0\right) \in F_{3}$ and $\left.T_{1}>0, \exists T_{2} \in\right] 0 ; T_{1}[$ such that $\Phi_{\sigma_{A}}\left(\sigma_{0}, T_{2}\right)>\underline{\sigma}, \frac{\partial \Phi_{\sigma_{A}}}{\partial t}\left(\sigma_{0}, T_{2}\right)=0$ and $\frac{\partial^{2} \Phi_{\sigma_{A}}}{\partial t^{2}}\left(\sigma_{0}, T_{2}\right) \leq 0$.

Hence, following the same development as Eq. 20, Eq. 21 and Eq. 22,

$$
\bar{u} \leq c\left(\Phi_{\sigma_{A}}\left(\sigma_{0}, T_{2}\right), \Phi_{\sigma_{B}}\left(\sigma_{0}, T_{2}\right)\right) .
$$

We recall that $\hat{\sigma}\left(\sigma_{A}\right)=\min \left\{\sigma_{B} \in\left[\sigma_{A} ; 1-\sigma_{A}\right] \mid \forall \sigma \geq \sigma_{B}, c\left(\sigma_{A}, \sigma\right) \leq \bar{u}\right\}$. Let $\hat{s}\left(\sigma_{A}\right)=s_{0}\left(\sigma_{A}, \hat{\sigma}\left(\sigma_{A}\right)\right)$. $\hat{s}$ decreases with $\sigma_{A}$ as illustrated in Fig. 3(b). So, $\hat{s}\left(\Phi_{\sigma_{A}}\left(\sigma_{0}, T_{2}\right)\right) \leq \hat{s}(\underline{\sigma})$. Moreover, since Eq. 27, $\Phi_{\sigma_{B}}\left(\sigma_{0}, T_{2}\right) \leq \hat{\sigma}\left(\Phi_{\sigma_{A}}\left(\sigma_{0}, T_{2}\right)\right)$ and $\Phi_{s}\left(\sigma_{0}, T_{2}\right) \leq \hat{s}\left(\Phi_{\sigma_{A}}\left(\sigma_{0}, T_{2}\right)\right)$ since $s_{0}\left(\Phi_{\sigma_{A}}\left(\sigma_{0}, T_{2}\right),.\right)$ is increasing. Moreover, $\frac{\partial \Phi_{s}}{\partial t}=-\bar{u}$, then $\Phi_{s}\left(\sigma_{0}, T_{1}\right)<\hat{s}(\underline{\sigma})$.

Finally, $\Phi_{\sigma_{B}}\left(\sigma_{0}, T_{1}\right)<\hat{\sigma}$ since $s_{0}(\underline{\sigma},$.$) is increasing. And \frac{\partial^{2} \Phi_{\sigma_{A}}}{\partial t^{2}}\left(\sigma_{0}, T_{1}\right) \geq 0$, that is $\bar{u} \geq c\left(\Phi_{\sigma_{A}}\left(\sigma_{0}, T_{1}\right)=\underline{\sigma}, \Phi_{\sigma_{B}}\left(\sigma_{0}, T_{1}\right)<\hat{\sigma}\right)$ which contradicts the assumption $(\mathrm{H} 2)$.

Figure 8 displays a 3D plot of the intersection between $K$ and the surface $\{\Phi(\sigma, t) \mid(\sigma, t) \in[\hat{\sigma}, 1-\underline{\sigma}] \times[0, T(\sigma)]\}$.

\subsubsection{Case 3: $\hat{\sigma}>\underline{\sigma}$ and (H3) there exists $\tilde{\sigma}, \underline{\sigma}<\tilde{\sigma}<\hat{\sigma}$, such that $\forall \sigma_{B} \in[\underline{\sigma}, \tilde{\sigma}], c\left(\underline{\sigma}, \sigma_{B}\right) \leq \bar{u}$}

On the contrary to case 2, with the assumption (H3), there may exist $\left.\sigma_{d} \in\right] \hat{\sigma} ; 1-\underline{\sigma}\left[\right.$ and $\left.\sigma_{e} \in\right] \underline{\sigma}, \tilde{\sigma}\left[\right.$ such that $\Phi\left(\sigma_{d}, T_{1}\right)=\left(\underline{\sigma}, \sigma_{e}, s_{0}\left(\underline{\sigma}, \sigma_{e}\right)\right)$ with $0<T_{1}<T\left(\sigma_{d}\right)$. The assumption of lemma 2 is not satisfied at $\sigma_{d}$ and $T(\sigma)$ and $\Phi(\sigma, T(\sigma))$ are not continuous at $\sigma_{d}: \lim _{\sigma \rightarrow \sigma_{d}-} \Phi(\sigma, T(\sigma)) \in \stackrel{\circ}{F}_{3}$, but $\Phi\left(\sigma_{d}, T\left(\sigma_{d}\right)\right) \in F_{4} \cup F_{0}$.

To complete the surface, we then have to extend the definition domain of $\Phi:\left[\underline{\sigma} ; \sigma_{e}\left[\cup[\hat{\sigma} ; 1-\underline{\sigma}] \times \mathbb{R}^{+} \rightarrow \mathbb{R}^{3}\right.\right.$ as illustrated in Figure 9. Especially, Figure 9 (b) shows the intersection between $\Phi(\sigma, t)$ and $F_{4}$ with the noticeable values $\sigma_{d}$ and $\sigma_{e}$. 


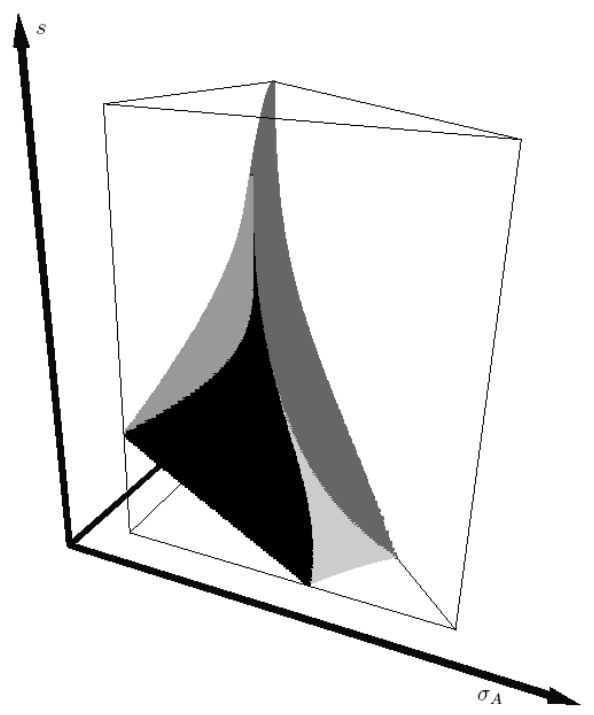

(a)

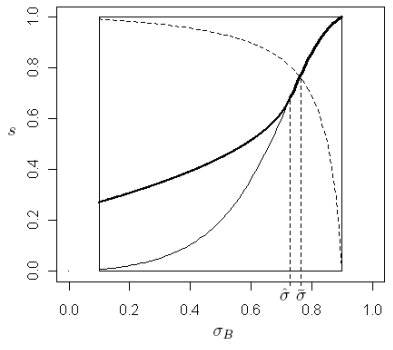

(b)

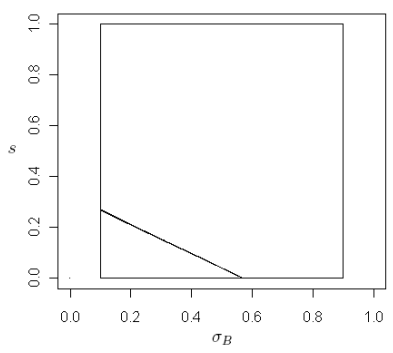

(c)

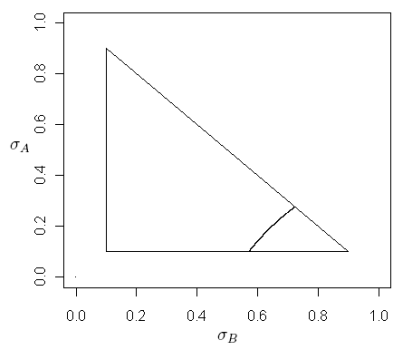

(d)

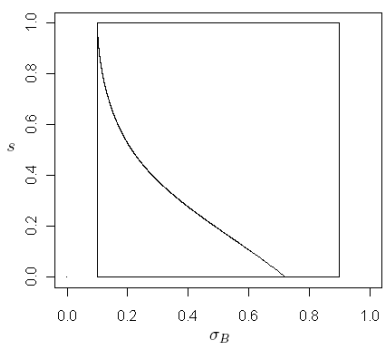

(e)

Figure 8: 8(a) The Euler approximation of $\{\Phi(\sigma, t) \mid \sigma \in[\hat{\sigma}, 1-\underline{\sigma}] \times[0, T(\sigma)]\}$ for $\underline{\sigma}=0.1$ and $\bar{u}=0.06$. The area $\left\{\Phi(\sigma, t) \mid \Phi(\sigma, T(\sigma)) \in F_{3}\right\}$ is colored gray. The area $\left\{\Phi(\sigma, t) \mid \Phi(\sigma, T(\sigma)) \in F_{4}\right\}$ is colored black. The area $\left\{\Phi(\sigma, t) \mid \Phi(\sigma, T(\sigma)) \in F_{0}\right\}$ is colored light gray. The area $\left\{\Phi(\sigma, t) \mid \Phi(\sigma, T(\sigma)) \in F_{2}\right\}$ is colored dark gray. 8(b), 8(c), 8(d), 8(e), the intersection between $\Phi(\sigma, t)$ and $F_{3}, F_{4}, F_{0}$ and $F_{2}$.

\subsection{Defining the viability domain inside the constraint set}

We denote

$$
D_{N_{0}}:=\left\{\Phi(\sigma, t) \mid \sigma \in\left[\underline{\sigma} ; \sigma_{e}\right] \cup[\hat{\sigma} ; 1-\underline{\sigma}], t \in[0 ; T(\sigma)]\right\}
$$

We denote $D_{N_{1}}$ the symmetric of $D_{N_{0}}$ by the transformation $\left(\sigma_{A}, \sigma_{B}, s\right) \rightarrow$ $\left(\sigma_{B}, \sigma_{A}, 1-s\right)$. 


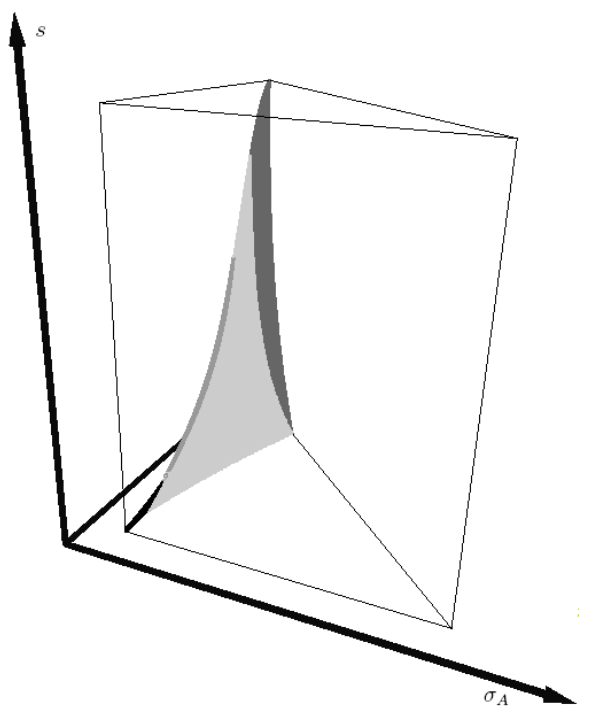

(a)

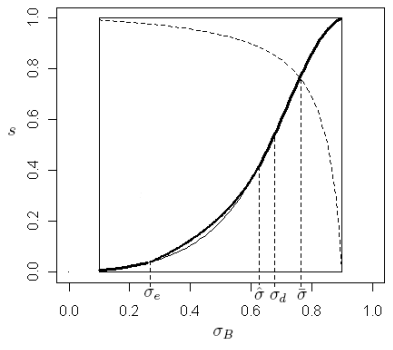

(b)

Figure 9: 9(a) The Euler approximation of $\left\{\Phi(\sigma, t) \mid \sigma \in\left[\underline{\sigma} ; \sigma_{e}[\cup[\hat{\sigma}, 1-\underline{\sigma}] \times[0, T(\sigma)]\}\right.\right.$ for $\underline{\sigma}=0.1$ and $\bar{u}=0.28$. The area $\left\{\Phi(\sigma, t) \mid \sigma \in\left[\underline{\sigma} ; \sigma_{e}[\}\right.\right.$ is colored black. The area $\{\Phi(\sigma, t) \mid \sigma \in$ $\left.[\hat{\sigma} ; 1-\underline{\sigma}] \Phi(\sigma, T(\sigma)) \in F_{3}\right\}$ is colored gray. The area $\left\{\Phi(\sigma, t) \mid \sigma \in[\hat{\sigma} ; 1-\underline{\sigma}] \Phi(\sigma, T(\sigma)) \in F_{0}\right\}$ is colored light gray. The area $\left\{\Phi(\sigma, t) \mid \sigma \in[\hat{\sigma} ; 1-\underline{\sigma}] \Phi(\sigma, T(\sigma)) \in F_{2}\right\}$ is colored dark gray. 9(b) displays the intersection between $\Phi(\sigma, t)$ and $F_{3}$.

\section{Remark 3.}

$$
D_{N_{1}}:=\left\{\bar{\Phi}(\sigma, t) \mid \sigma \in\left[\underline{\sigma} ; \sigma_{e}\right] \cup[\hat{\sigma} ; 1-\underline{\sigma}], t \in[0 ; \bar{T}(\sigma)]\right\}
$$

where $\bar{\Phi}(\sigma, t):\left[\underline{\sigma} ; \sigma_{e}\right] \cup[\hat{\sigma} ; 1-\underline{\sigma}] \times\left[0 ;+\infty\left[\rightarrow \mathbb{R}^{3}\right.\right.$ such that

$$
\left\{\begin{array}{l}
\bar{\Phi}(\sigma, 0)=\left(\sigma, \underline{\sigma}, 1-s_{0}(\underline{\sigma}, \sigma)\right) \\
\frac{\partial \bar{\Phi}(\sigma, t)}{\partial t}=\left(-f_{1}(\bar{\Phi}(\sigma, t)),-f_{2}(\bar{\Phi}(\sigma, t)), \bar{u}\right) .
\end{array}\right.
$$

and $\bar{T}(\sigma)=\max \{T \mid \forall t \in[0 ; T], \bar{\Phi}(\sigma, t) \in K\}$.

$D_{N_{0}}$ separates $K$ into two subsets. We denote

$$
\begin{aligned}
\bar{D}:=\{x \in K \mid & \exists x(.) \text { continuous }:[0,1] \rightarrow K, x(0)=x, x(1)=(1-\underline{\sigma}, \underline{\sigma}, 1), \\
& \forall t \in[0,1], x(t) \in K, \\
& \left.\{x(t) \mid t \in] 0,1]\} \cap D_{N_{1}}=\emptyset\right\} .
\end{aligned}
$$


In the same manner, $D_{N_{1}}$ separates $K$ into two subsets. We denote

$$
\begin{aligned}
\underline{D}:=\{x \in K \mid & \exists x(.) \text { continuous }:[0,1] \rightarrow K, x(0)=x, x(1)=(\underline{\sigma}, 1-\underline{\sigma}, 0), \\
& \forall t \in[0,1], x(t) \in K, \\
& \left.\{x(t) t \in] 0,1]\} \cap D_{N_{1}}=\emptyset\right\}
\end{aligned}
$$

Let $D \subset K$ defined by

$$
D:=\bar{D} \cap \underline{D}
$$

Figure 10 displays different 3d-plots of such a subset $D$ when $\underline{\sigma}=0.1$ and $\bar{u}=0.06$.

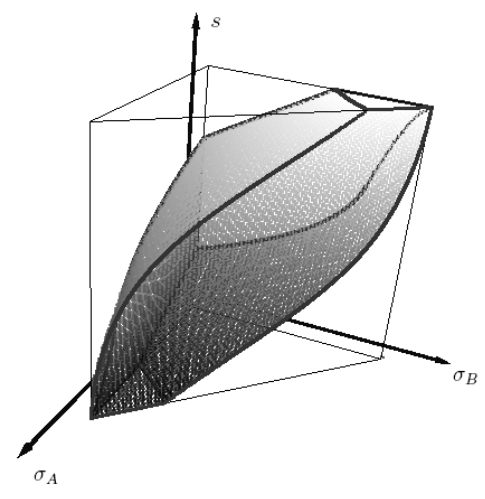

(a)

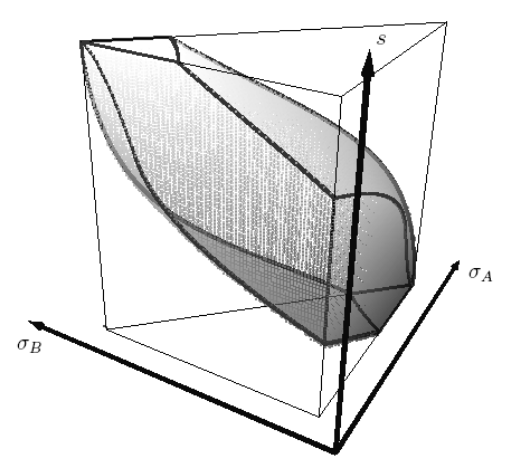

(c)

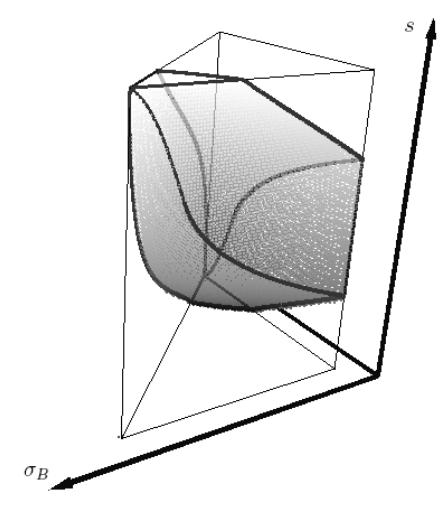

(b)

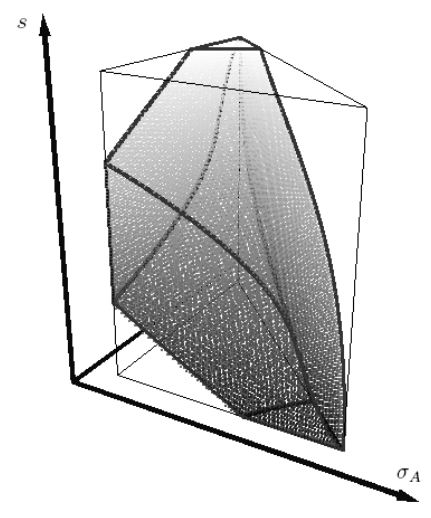

(d)

Figure 10: Four different views of the 3D-viability kernel for $\underline{\sigma}=0.1$ and $\bar{u}=0.06$. 
Theorem 2. $D$ is a viability domain under $F$.

Proof. We denote $D_{F_{0}}:=D \cap F_{0}, D_{F_{1}}:=D \cap F_{1}, D_{F_{2}}:=D \cap F_{2}, D_{F_{3}}:=$ $D \cap F_{3}$, and $D_{F_{4}}:=D \cap F_{4}$. The boundary of $D, \partial D$ equals :

$$
\partial D=D_{F_{0}} \cup D_{F_{1}} \cup D_{F_{2}} \cup D_{F_{3}} \cup D_{F_{4}} \cup D_{N_{0}} \cup D_{N_{1}} \text {. }
$$

- If $\left(\sigma_{A}, \sigma_{B}, s\right) \in D_{F_{0}}^{\circ}, T_{D}\left(\sigma_{A}, \sigma_{B}, s\right)=\mathbb{R}^{2} \times \mathbb{R}^{+}$, so $f\left(\sigma_{A}, \sigma_{B}, s, u\right) \in$ $T_{D}\left(\sigma_{A}, \sigma_{B}, s\right)$ for $u \in[0, \bar{u}]$.

- If $\left(\sigma_{A}, \sigma_{B}, s\right) \in D_{F_{1}}^{\circ}, T_{D}\left(\sigma_{A}, \sigma_{B}, s\right)=\mathbb{R}^{2} \times \mathbb{R}^{-}$, so $f\left(\sigma_{A}, \sigma_{B}, s, u\right) \in$ $T_{D}\left(\sigma_{A}, \sigma_{B}, s\right)$ for $u \in[-\bar{u}, 0]$.

- If $\left(\sigma_{A}, \sigma_{B}, s\right) \in \stackrel{\circ}{D_{F_{2}}}, T_{D}\left(\sigma_{A}, \sigma_{B}, s\right)=\{(x, y, z) \mid x+y \leq 0\}$, so $f\left(\sigma_{A}, \sigma_{B}, s, u\right) \in$ $T_{D}\left(\sigma_{A}, \sigma_{B}, s\right)$ for $u \in U$.

- If $\left(\sigma_{A}, \sigma_{B}, s\right) \in D_{F_{3}}^{\circ}, T_{D}\left(\sigma_{A}, \sigma_{B}, s\right)=\mathbb{R}^{+} \times \mathbb{R}^{2}$, and $f_{1}\left(\sigma_{A}, \sigma_{B}, s\right)>0$, so $f\left(\sigma_{A}, \sigma_{B}, s, u\right) \in T_{D}\left(\sigma_{A}, \sigma_{B}, s\right)$ for $u \in U$.

- We have the same, if $\left(\sigma_{A}, \sigma_{B}, s\right) \in{\stackrel{\circ}{F_{4}}}, f\left(\sigma_{A}, \sigma_{B}, s, u\right) \in T_{D}\left(\sigma_{A}, \sigma_{B}, s\right)$ for $u \in U$.

- If $\left(\sigma_{A}, \sigma_{B}, s\right) \in D_{N_{0}}^{\circ}, f\left(\sigma_{A}, \sigma_{B}, s, u\right) \in T_{D}\left(\sigma_{A}, \sigma_{B}, s\right)$ for $u=\bar{u}$.

- If $\left(\sigma_{A}, \sigma_{B}, s\right) \in D_{N_{1}}^{\circ}, f\left(\sigma_{A}, \sigma_{B}, s, u\right) \in T_{D}\left(\sigma_{A}, \sigma_{B}, s\right)$ for $u=-\bar{u}$.

Moreover,

- If $\left(\sigma_{A}, \sigma_{B}, s\right) \in \widehat{D_{F_{0}} \cap D_{F_{2}}}, T_{D}\left(\sigma_{A}, \sigma_{B}, s\right)=\{(x, y, z) \mid x+y \leq 0, z \geq 0\}$, so $f\left(\sigma_{A}, \sigma_{B}, s, u\right) \in T_{D}\left(\sigma_{A}, \sigma_{B}, s\right)$ for $u \in[0, \bar{u}]$ since $\left(f_{1}\left(\sigma_{A}, \sigma_{B}, s\right)+\right.$ $\left.f_{2}\left(\sigma_{A}, \sigma_{B}, s\right)\right) \leq 0$ when $\left(\sigma_{A}+\sigma_{B}\right)=1$.

- If $\left(\sigma_{A}, \sigma_{B}, s\right) \in \widehat{D_{F_{0}} \cap D_{F_{4}}}, T_{D}\left(\sigma_{A}, \sigma_{B}, s\right)=\mathbb{R}^{2} \times \mathbb{R}^{+}$, so $f\left(\sigma_{A}, \sigma_{B}, s, u\right) \in$ $T_{D}\left(\sigma_{A}, \sigma_{B}, s\right)$ for $u \in[0, \bar{u}]$.

- If $\left(\sigma_{A}, \sigma_{B}, s\right) \in D_{F_{0}} \cap D_{N_{0}}, f\left(\sigma_{A}, \sigma_{B}, s, u\right) \in T_{D}\left(\sigma_{A}, \sigma_{B}, s\right)$ for $u=\bar{u}$.

- If $\left(\sigma_{A}, \sigma_{B}, s\right) \in \widehat{D}_{F 3 \cap D_{F 4}}, T_{D}\left(\sigma_{A}, \sigma_{B}, s\right)=\mathbb{R}^{+} \times \mathbb{R}^{+} \times \mathbb{R}$, so $f\left(\sigma_{A}, \sigma_{B}, s, u\right) \in$ $T_{D}\left(\sigma_{A}, \sigma_{B}, s\right)$ for $u \in U$ since $f_{1}\left(\sigma_{A}, \sigma_{B}, s\right)>0$ and $f_{2}\left(\sigma_{A}, \sigma_{B}, s\right)>0$.

- If $\left(\sigma_{A}, \sigma_{B}, s\right) \in \widehat{D F 4}_{N_{0} \cap D_{0}}, f\left(\sigma_{A}, \sigma_{B}, s, u\right) \in T_{D}\left(\sigma_{A}, \sigma_{B}, s\right)$ for $u=\bar{u}$. 
- If $\left(\sigma_{A}, \sigma_{B}, s\right) \in \widehat{D}_{F_{2} \cap D_{N_{0}}}, f\left(\sigma_{A}, \sigma_{B}, s, u\right) \in T_{D}\left(\sigma_{A}, \sigma_{B}, s\right)$ for $u=\bar{u}$.

- If $\left(\sigma_{A}, \sigma_{B}, s\right) \in D_{F_{3}} \cap D_{N_{0}}$,

- if $\sigma_{B} \in\left[\underline{\sigma}, \sigma_{e}\left[, f\left(\sigma_{A}, \sigma_{B}, s, u\right) \in T_{D}\left(\sigma_{A}, \sigma_{B}, s\right)\right.\right.$ for $u \in\left[c\left(\underline{\sigma}, \sigma_{B}\right), \bar{u}\right]$.

- if $\sigma_{B} \in\left[\sigma_{e}, \hat{\sigma}\right], f\left(\sigma_{A}, \sigma_{B}, s, u\right) \in T_{D}\left(\sigma_{A}, \sigma_{B}, s\right)$ for $u=\bar{u}$

- if $\sigma_{B} \in\left[\hat{\sigma}, 1-\underline{\sigma}\left[, f\left(\sigma_{A}, \sigma_{B}, s, u\right) \in T_{D}\left(\sigma_{A}, \sigma_{B}, s\right)\right.\right.$ for $u \in\left[c\left(\underline{\sigma}, \sigma_{B}\right), \bar{u}\right]$.

- and $f(\underline{\sigma}, 1-\underline{\sigma}, 1, u) \in T_{D}(\underline{\sigma}, 1-\underline{\sigma}, 1)$ for $u \in\left[c\left(\underline{\sigma}, \sigma_{B}\right), 0\right]$.

The proof of the non emptiness of the intersection between the contingent cone and the dynamics on the other points of the boundary of $D$ can be deduced from the problem symmetry by the transformation $\left(\sigma_{A}, \sigma_{B}, s\right) \rightarrow$ $\left(\sigma_{B}, \sigma_{A}, 1-s\right)$.

\section{Control strategy using the viability domain}

\subsection{The regulation map}

$D$ is a viability domain. Consequently, it allows to build a regulation map, $R_{D}$, with non empty values on $D$. That means that any $x \in D$ is viable. Moreover, there exists a viable evolution governed by the differential inclusion associated with the regumation map (theorem 1).

The regulation map is directly defined from the intersection between the dynamics and the contingent cone of $D$ (Def. 4):

$$
\forall x \in D, R_{D}(x):=\left\{u \in U(x) \mid f(x, u) \in T_{D}(x)\right\} .
$$

Let :

$$
\begin{aligned}
& \underline{D_{N_{0}}}:=D_{N_{0}}-\left\{\Phi(\sigma, 0) \mid \sigma \in\left[\underline{\sigma} ; \sigma_{e}[\cup[\hat{\sigma} ; 1-\underline{\sigma}]\}\right.\right. \\
& \underline{D_{N 1}}:=D_{N_{1}}-\left\{\bar{\Phi}_{1}(\sigma, 0) \mid \sigma \in\left[\underline{\sigma} ; \sigma_{e}[\cup[\hat{\sigma} ; 1-\underline{\sigma}]\}\right.\right.
\end{aligned}
$$

From the proof of theorem 2,

- for $x \in \underline{D_{N_{0}}}, R_{D}(x)=\bar{u}$; for $x \in \underline{D_{N 1}}, R_{D}(x)=-\bar{u}$

- for $x \in D_{F 0}^{\circ} \cup\left(\widehat{D_{F 0} \cap D_{F 2}}\right) \cup\left(\widehat{D_{F 0} \cap D_{F 4}}\right), R_{D}(x)=[0 ; \bar{u}]$

- for $x \in D_{F 1}^{\circ} \cup\left(\widehat{D_{F 1} \cap D_{F 2}}\right) \cup\left(\widehat{D_{F 1} \cap D_{F 3}}\right), R_{D}(x)=[-\bar{u} ; 0]$ 
- for $\left(\underline{\sigma}, \sigma_{B}, s\right) \in D_{N_{0}}-\left(\underline{D_{N_{0}}} \cup\{(\underline{\sigma}, 1-\underline{\sigma}, 1)\}\right), R_{D}\left(\underline{\sigma}, \sigma_{B}, s\right)=\left[c\left(\underline{\sigma}, \sigma_{B}\right), \bar{u}\right]$

- for $\left(\sigma_{A}, \underline{\sigma}, s\right) \in D_{N_{1}}-\left(\underline{D_{N 1}} \cup\{(1-\underline{\sigma}, \underline{\sigma}, 0)\}\right), R_{D}\left(\sigma_{A}, \underline{\sigma}, s\right)=\left[-\bar{u},-c\left(\underline{\sigma}, \sigma_{A}\right)\right]$

- $R_{D}(\underline{\sigma}, 1-\underline{\sigma}, 1)=\left[c\left(\underline{\sigma}, \sigma_{B}\right), 0\right]$

- $R_{D}(1-\underline{\sigma}, \underline{\sigma}, 0)=\left[0,-c\left(\underline{\sigma}, \sigma_{B}\right)\right]$

- for $x \in D-D_{N_{0}} \cup D_{N_{1}} \cup D_{F_{0}} \cup D_{F 1}, R_{D}(x)=U$.

\subsection{Viable evolutions}

From theorem 1, any control function regulating a viable solution $x($.$) in$ $D$ obeys the regulation law

$$
\text { for almost all t, } u(t) \in R_{D}(x(t)) \text {. }
$$

That means that thanks to this regulation map, if the present situation lies in the viability domain, we can control the system to remain in the constraint set, and therefore preserve coexistence of both monolingual groups. At each time, there may be several controls that ensure viability. The next issue that arises is the choice of an effective univocal control function.

\subsection{Slow viable evolutions}

From a political viewpoint, the strategy that minimises control strength at each time may be attractive since it reduces the strength of the measures to carry out. We derive from the regulation map such a strategy below.

\subsubsection{Minimal selection of the regulation map}

We first consider the minimal selection, $R_{D}^{*}$, of the regulation map $R_{D}$. Actually, the values of the regulation map $R_{D}$ are closed and convex, so we can associate with $x \in D$ the viable control with minimal norm :

$$
\begin{aligned}
R_{D}^{*}(x) & :=m\left(R_{D}(x)\right) \\
& :=\left\{u \in R_{D}(x)\|\| u\left\|=\min _{y \in R_{D}(x)}\right\| y \|\right\}
\end{aligned}
$$

$R_{D}^{*}$ is then defined on $D$ by :

- for $x \in \underline{D_{N 0}}, R_{D}^{*}(x)=\bar{u} ;$ for $x \in \underline{D_{N 1}}, R_{D}^{*}(x)=-\bar{u}$

- for $x \in D_{F_{0}}^{\circ} \cup\left(D_{F 0} \stackrel{\circ}{\cap} D_{F 2}\right) \cup\left(D_{F 0} \stackrel{\circ}{\cap} D_{F 4}\right), R_{D}^{*}(x)=\{0\}$ 
- for $x \in D_{F 1}^{\circ} \cup\left(D_{F 1} \stackrel{\circ}{\cap} D_{F 2}\right) \cup\left(D_{F 1} \stackrel{\circ}{\cap} D_{F 3}\right), R_{D}^{*}(x)=\{0\}$

- for $\left(\underline{\sigma}, \sigma_{B}, s\right) \in D_{N_{0}}-\left(\underline{D_{N_{0}}} \cup\{(\underline{\sigma}, 1-\underline{\sigma}, 1)\}\right), R_{D}^{*}\left(\underline{\sigma}, \sigma_{B}, s\right)=\left\{\max \left(0, c\left(\underline{\sigma}, \sigma_{B}\right)\right)\right\}$

- for $\left(\sigma_{A}, \underline{\sigma}, s\right) \in D_{N 1}-\left(\underline{D_{N 1}} \cup\{(1-\underline{\sigma}, \underline{\sigma}, 0)\}\right), R_{D}^{*}\left(\sigma_{A}, \underline{\sigma}, s\right)=\left\{\min \left(-c\left(\underline{\sigma}, \sigma_{A}\right), 0\right)\right\}$

- $R_{D}^{*}(\underline{\sigma}, 1-\underline{\sigma}, 1)=\{0\}$

- $R_{D}^{*}(1-\underline{\sigma}, \underline{\sigma}, 0)=\{0\}$

- for $x \in D-D_{N_{0}} \cup D_{N_{1}} \cup D_{F_{0}} \cup D_{F 1}, R_{D}^{*}(x)=\{0\}$.

Theorem 3. For any initial state $x_{0} \in D$, there exists a viable solution starting at $x_{0}$ which is regulated by $R_{D}^{*}$ in the sense that $\left\{\begin{array}{l}\text { for almost all } t \geq 0, \\ u(t) \in R_{D}^{*}(x(t))\end{array}\right.$

ProOF. Let $\hat{R}_{D}$ the set-valued map defined by

- for $x \in \underline{D_{N 0}}, \hat{R}_{D}(x)=[0 ; \bar{u}]$

- for $x \in \underline{D_{N 1}}, \hat{R}_{D}(x)=[-\bar{u} ; 0]$

- for $x \in D_{F 0}^{\circ} \cup\left(D_{F_{0}} \stackrel{\circ}{\cap} D_{F 2}\right) \cup\left(D_{F_{0}} \stackrel{\circ}{\cap} D_{F 4}\right), \hat{R}_{D}(x)=\{0\}$

- for $x \in D_{F 1}^{\circ} \cup\left(D_{F 1} \stackrel{\circ}{\cap} D_{F 2}\right) \cup\left(D_{F 1} \stackrel{\circ}{\cap} D_{F 3}\right), \hat{R}_{D}(x)=\{0\}$

- for $\left(\underline{\sigma}, \sigma_{B}, s\right) \in D_{N_{0}}-\left(\underline{D_{N_{0}}} \cup\{(\underline{\sigma}, 1-\underline{\sigma}, 1)\}\right), \hat{R}_{D}\left(\underline{\sigma}, \sigma_{B}, s\right)=[0 ; \bar{u}]$

- for $\left(\sigma_{A}, \underline{\sigma}, s\right) \in D_{N 1}-\left(\underline{D_{N 1}} \cup\{(1-\underline{\sigma}, \underline{\sigma}, 0)\}\right), \hat{R}_{D}\left(\sigma_{A}, \underline{\sigma}, s\right)=[-\bar{u} ; 0]$

- $\hat{R}_{D}(\underline{\sigma}, 1-\underline{\sigma}, 1)=[0 ; \bar{u}]$

- $\hat{R}_{D}(1-\underline{\sigma}, \underline{\sigma}, 0)=[-\bar{u} ; 0]$

- for $x \in D-D_{N_{0}} \cup D_{N_{1}} \cup D_{F_{0}} \cup D_{F 1}, \hat{R}_{D}(x)=\{0\}$. 
$\hat{R}_{D}$ is a selection of $F$ (Def. 5 ) with convex values. So, from theorem 4 in Appendix A, for any initial state $x_{0} \in D$, there exists a viable solution to control system (7) starting at $x_{0}$ which is regulated by the selection $S\left(\hat{R}_{D}\right)$ of the regulation map $R_{D}$, in the sense that

$$
\left\{\begin{array}{l}
\text { for almost all } t \geq 0 \\
u(t) \in S\left(\hat{R}_{D}\right)(x(t)):=R_{D}(x(t)) \cap \hat{R}_{D}(x(t))
\end{array}\right.
$$

$S\left(\hat{R}_{D}\right)(x)$ is defined by

- for $x \in \underline{D_{N 0}}, S\left(\hat{R}_{D}\right)(x)=\bar{u}$; for $x \in \underline{D_{N 1}}, S\left(\hat{R}_{D}\right)(x)=-\bar{u}$

- for $x \in D_{F_{0}}^{\circ} \cup\left(D_{F_{0}} \stackrel{\circ}{\cap} D_{F_{2}}\right) \cup\left(D_{F_{0}} \stackrel{\circ}{\cap} D_{F 4}\right), S\left(\hat{R}_{D}\right)(x)=\{0\}$

- for $x \in D_{F 1}^{\circ} \cup\left(D_{F 1} \stackrel{\circ}{\cap} D_{F 2}\right) \cup\left(D_{F 1} \stackrel{\circ}{\cap} D_{F 3}\right), S\left(\hat{R}_{D}\right)(x)=\{0\}$

- for $\left(\underline{\sigma}, \sigma_{B}, s\right) \in D_{N 0}-\left(D_{N 0} \cup\{(\underline{\sigma}, 1-\underline{\sigma}, 1)\}\right), S\left(\hat{R}_{D}\right)\left(\underline{\sigma}, \sigma_{B}, s\right)=$ $\left[\max \left(c\left(\underline{\sigma}, \sigma_{B}\right), 0\right) ; \bar{u}\right]$

- for $\left(\sigma_{A}, \underline{\sigma}, s\right) \in D_{N 1}-\left(D_{N 1} \cup\{(1-\underline{\sigma}, \underline{\sigma}, 0)\}\right), S\left(\hat{R}_{D}\right)\left(\sigma_{A}, \underline{\sigma}, s\right)=$ $\left[-\bar{u} ; \min \left(-c\left(\underline{\sigma}, \sigma_{A}\right), 0\right)\right]$

- $S\left(\hat{R}_{D}\right)(\underline{\sigma}, 1-\underline{\sigma}, 1)=\{0\}$

- $S\left(\hat{R}_{D}\right)(1-\underline{\sigma}, \underline{\sigma}, 0)=\{0\}$

- for $x \in D-D_{N_{0}} \cup D_{N_{1}} \cup D_{F 0} \cup D_{F 1}, S\left(\hat{R}_{D}\right)(x)=\{0\}$.

$S\left(\hat{R}_{D}\right)$ only differs from $R_{D}^{*}$ for $\left(\underline{\sigma}, \sigma_{B}, s\right) \in D_{N_{0}}-\left(\underline{D_{N_{0}}} \cup\{(\underline{\sigma}, 1-\underline{\sigma}, 1)\}\right)$ and for the symmetric.

Assume that $x_{0}=\left(\underline{\sigma}, \sigma_{B}, s\right) \in N_{D_{0}}-\left(N_{D_{0}} \cup\{(\underline{\sigma}, 1-\underline{\sigma}, 1)\}\right)$.

Let $x($.$) be a viable evolution starting at x_{0}$ and regulated by $S\left(\hat{R}_{D}\right): u(0) \in$ $\left[\max \left(c\left(\underline{\sigma}, \sigma_{B}\right), 0\right) ; \bar{u}\right]$.

- if $c\left(\underline{\sigma}, \sigma_{B}\right) \leq 0, u(0) \in[0 ; \bar{u}]$ and $\exists \delta>0$ such that $\left.\forall t \in\right] 0 ; \delta[, x(t) \in \stackrel{\circ}{D}$ and for almost all $t \in\left[0, \delta\left[, u(t)=\{0\} \in R_{D}^{*}(x(t))\right.\right.$

- if $c\left(\underline{\sigma}, \sigma_{B}\right)>0, \exists \delta>0$ such that $\forall t \in\left[0 ; \delta\left[, x(t) \in \underline{N_{D 0}}\right.\right.$, so for almost all $t \in\left[0 ; \delta\left[, u(t)=c\left(\underline{\sigma}, \sigma_{B}(t)\right) \in R_{D}^{*}(x(t))\right.\right.$.

Consequently, for any initial state $x_{0} \in D$, there exists a viable solution to control system (7) starting at $x_{0}$ which is regulated by the minimal selection $R^{*}{ }_{D}$. 


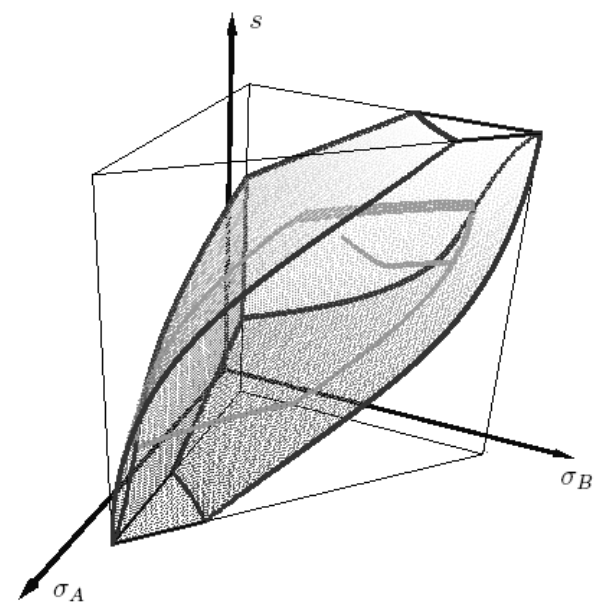

(a)

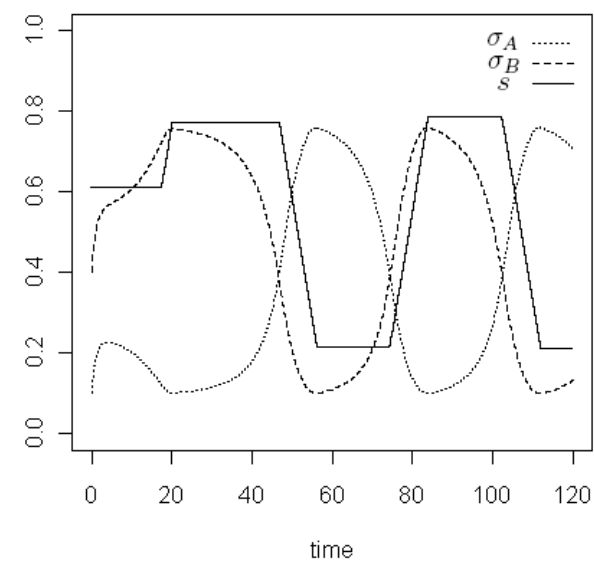

(b)

Figure 11: An example of slow viable evolution : the 3D-plot of its trajectory 11(a) and the evolution of the three variable values over time $11(\mathrm{~b})(\underline{\sigma}=0.1$ and $\bar{u}=0.06)$.

\subsection{The computation of a particular slow viable evolution}

The slow viable evolution consists in choosing at each time the control with minimal norm. In the case of the language competition model, the control is the variation speed of the relative prestige of both languages. Consequently, the slow viable evolution exhibits constant relative prestige periods until viability is at stake. For instance, in the case of the slow viable evolution starting with $10 \%$ of monolingual speakers of language $A, 40 \%$ of monolingual speakers of language $B$ (and consequently $50 \%$ of bilingual speakers), described in figure 11, the relative prestige remains constant between $t=0$ and $t=17.5, t=20.2$ and $t=47.0, t=56.3$ and $t=74.4$, and $t=84.1$ and $t=102.3$. However, these constant relative prestige periods are separated by prestige variation periods. Actually, a constant prestige evolution would lead to exit the viability domain (remark 1). The cumulative length of the variation prestige periods during this simulation represents $26 \%$ of the full simulation length.

It is also worth noting that to ensure coexistence prestige variation politics have to be undertaken when the constant prestige evolution reaches the boundary of the viability domain. That means that measures may have to be undertaken relatively far from the constraint set boundary : for instance, at 
time $t=47.0$, measures to increase the relative prestige of language $B$ have to be undertaken to prevent language $B$ community to go below the given threshold, but at that time, both communities size are far this threshold: $\sigma_{A}=41 \%$ and $\sigma_{B}=37 \%$. This illustrates the viability analysis as a tool of anticipation to take measures to prevent future viability loss.

\section{Conclusion}

Abrams and Strogatz [2] end their paper of the analysis of their model of language competition by the statement that " Contrary to the models stark prediction, bilingual societies do, in fact, exist. [...] The example of Quebec French demonstrates that language decline can be slowed by strategies such as policy-making, education and advertising, in essence increasing an endangered languages status."' Following this way, we have considered the status, the prestige, as a variable in a model of language competition taking explicitly into account the bilingual subpopulation. Crystal [1] describes the main mechanisms that make the prestige vary. We do not go into this detail but assume that the variations of this prestige with time are bounded (policy making as education take time).

We have then answered the question of determining a set of prestige variations that preserve both monolingual subpopulations following a viability theory approach.

- we have defined in the state space the constraint set representing the preservation of both monolingual subpopulations

- this constraint set is not a viability domain, so we have built inside it a viability domain : from all states of a viability domain there exists a control such that the evolution remains in the viability domain. This domain is a true set where both monolingual subpopulations can be preserved

- we have then proposed a selection of the regulation map that governs slow viable evolutions

- finally, we have illustrated this method from a given state, showing how a slow viable evolution made of constant control periods separated by specified interventions allow to preserve both monolingual subpopulations, although constant policy would lead to the extinction of one of them. 


\section{Appendix A. Selection of viable solutions : a definition and a the- orem}

Definition 5 (Selection Procedure). Let $Y$ a normed space. A selection procedure of a set-valued map $F: X \rightsquigarrow Y$ is a set-valued map $S_{F}: X \rightsquigarrow Y$ satisfying

$\left\{(i) \quad \forall x \in \operatorname{Dom}(F), S(F(x)):=S_{F}(x) \cap F(x) \neq \emptyset\right.$

( (ii) the graph of $S_{F}$ is closed

The set-valued map $S(F): x \rightsquigarrow S(F(x))$ is called the selection of $F$.

Theorem 4. Let us consider a Marchaud control system $(U, f)$ and suppose that $K$ is a viability domain. Let $S_{R_{K}}$ be a selection of the regulation map $R_{K}$. Suppose that the values of $S_{R_{K}}$ are convex. Then, for any initial state $x_{0} \in K$, there exists a viable solution starting at $x_{0}$ and a viable control to control system $(U, f)$ which are regulated by the selection $S\left(R_{K}\right)$ of the regulation map $R_{K}$, in the sense that $\left\{\begin{array}{l}\text { for almost all } t \geq 0, \\ u(t) \in S\left(R_{K}\right)(x(t)):=R_{K}(x(t)) \cap S_{R_{K}}(x(t))\end{array}\right.$ 
[1] D. Crystal, Language Death, Cambridge University Press, Cambridge, 2000 .

[2] D. Abrams, S. Strogatz, Modelling the dynamics of language death, Nature 424 (6951) (2003) 900.

[3] D. Crystal, The Cambridge Encyclopedia of Language, Cambridge University Press, Cambridge, 1987.

[4] I. Baggs, H. I. Freedman, A mathematical model for the dynamics of interactions between a unilingual and a bilingual population : Persistenece versus extinction, J. Math. Sociol. 16 (1) (1990) 51-75.

[5] J. Wyburn, J. Hayward, The future of bilingualism : An application of the baggs and freedman model, J. Math. Sociol. 32 (2008) 267-284.

[6] H. El-Owaidy, M. Ismail, A mathematical model of bilingualism, Appl. Math. Comput. 131 (2002) 415-432.

[7] J. Mira, A. Paredes, Interlinguistic similarity and language death dynamics, Europhys. Lett. 69 (2005) 1031-1034.

[8] X. Castello, V. Eguíluz, M. S. Miguel, Ordering dynamics with two nonexcluding options: bilingualism in language competition, New J. Phys. 8 (2006) 308-322.

[9] J. Minett, W. Wang, Modelling endangered languages: the effects of bilinguism and social structure, Lingua 118 (2008) 19-45.

[10] L. Chapel, X. Castellò, C. Bernard, G. Deffuant, V. M. Eguíluz, S. Martin, M. S. Miguel, Viability and resilience of languages in competition, PLoS ONE 5 (1) (2010) e8681. doi:10.1371/journal.pone.0008681.

[11] J. Aubin, Viability Theory, Birkhäuser, Basel, 1991.

[12] W.-Y. Wang, J. Minett, The invasion of language: emergence, change and death, Trends in Ecology and Evolution 20 (5) (2005) 263269. 